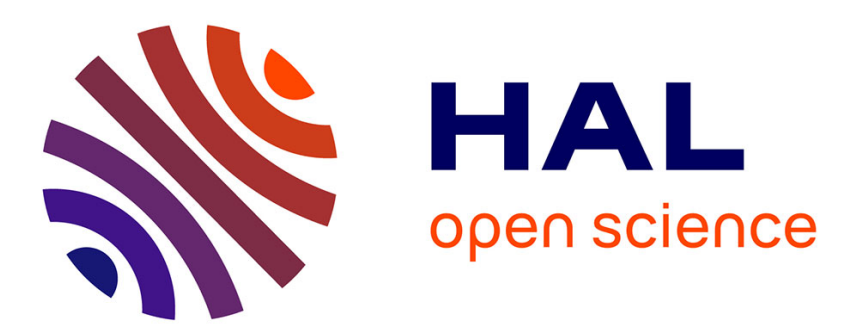

\title{
Sinusoïdal flow of blood in a cylindrical deformable vessel exposed to an external magnetic field
}

Agnès Drochon

\section{To cite this version:}

Agnès Drochon. Sinusoïdal flow of blood in a cylindrical deformable vessel exposed to an external magnetic field. European Physical Journal: Applied Physics, 2016, 10.1051/epjap/2016150530 . hal02063582

\section{HAL Id: hal-02063582 \\ https://hal.utc.fr/hal-02063582}

Submitted on 18 Feb 2021

HAL is a multi-disciplinary open access archive for the deposit and dissemination of scientific research documents, whether they are published or not. The documents may come from teaching and research institutions in France or abroad, or from public or private research centers.
L'archive ouverte pluridisciplinaire HAL, est destinée au dépôt et à la diffusion de documents scientifiques de niveau recherche, publiés ou non, émanant des établissements d'enseignement et de recherche français ou étrangers, des laboratoires publics ou privés. 


\section{EPJ AP \\ Applied Physics}

EPJ ors

Eur. Phys. J. Appl. Phys. (2016) 73: 31101

DOI: $10.1051 /$ epjap/2016150530

Sinusoïdal flow of blood in a cylindrical deformable vessel exposed to an external magnetic field

Agnès Drochon 


\title{
Sinusoïdal flow of blood in a cylindrical deformable vessel exposed to an external magnetic field
}

Agnès Drochon ${ }^{\mathrm{a}}$

Université de Technologie de Compiègne, Galileo Galilei Sorbonne Universités, UMR CNRS 7338, 60200 Compiègne, France

Received: 24 October 2015 / Received in final form: 12 February 2016 / Accepted: 16 February 2016 Published online: 22 March 2016 - (C) EDP Sciences 2016

\begin{abstract}
The present work provides an analytical solution for the sinusoïdal flow of blood in a cylindrical elastic vessel exposed to an external magnetic field. The vessel is supposed to have non-conducting walls and the induced electric and magnetic fields are neglected. In other words, the well-known calculation of Womersley is revisited through the inclusion of the Lorentz force in the Navier-Stokes equations. A dispersion equation is obtained. This equation admits two types of solutions: the Young waves (mainly associated with radial deformation of the vessel) and the Lamb waves (mainly associated with longitudinal displacements in the vessel wall). It is demonstrated that the external magnetic field has an influence on the wave celerities, on the fluid velocity profiles, and on the wall displacements. It tends to reduce the blood flow and flatten the velocity profile, in the case of Young waves. The pulsatile character of the flow is also dampened. However, these effects become detectable for high values of the Hartmann number $(M>4$, corresponding to $B_{0}>36 \mathrm{~T}$ with numerical data pertaining to large human arteries) and remain negligible in the context of magnetic resonance imaging $\left(B_{0} \leq 3 \mathrm{~T}\right.$, or even $\left.7 \mathrm{~T}\right)$.
\end{abstract}

\section{Context and objectives}

Several biomedical applications require a good knowledge of the physics of blood flow and pressure pulse propagation in a deformable vessel, in the presence of an external static magnetic field:

\section{i) Cardiac MRI and gating}

In the case of magnetic resonance imaging (MRI) of the heart, the charged particles of the blood get deflected by the Lorentz force, thus inducing electrical currents and voltages across the vessel wall and in the surrounding tissues. These voltages disturb the electrocardiogram (ECG) detected at the surface of the thorax, making the ECG-based image synchronization inaccurate. Several approaches have been proposed to correct the ECG signals for these magnetohydrodynamic (MHD) induced artifacts [1-4]. On the contrary, Kyriakou et al. [5], decided to try to use the MHD effect measured with ECG as a biomarker of cardiac output, since it is sensitive to magnetic flux density, flow orientation with respect to the magnetic field lines, and velocity of the blood. Other groups $[6,7]$ even propose to use the MHD induced voltage itself as a tool for cardiac synchronization.

Besides, the MHD induced voltages may also impact surrounding tissues (for example, the heart itself) and other vital signs. This point is addressed in a special issue

\footnotetext{
a e-mail: agnes.drochon@utc.fr
}

(no. 87) of Progress in Biophysics and Molecular Biology, 2005.

ii) MRI pulse wave velocity measurement

Pulse wave velocity (PWV) is known to be an indicator of arterial stiffness, and, as a matter of fact, a predictor of cardiovascular risk. Diseases such as atherosclerosis affect the human aortic wall in localized regions. The formation of plaque changes the mechanical properties of the wall. MRI has been proposed by several groups as a noninvasive tool to assess PWV [8-11].

\section{iii) Capture of pulsatile blood energy}

Quite recently, a new direction of research has emerged, aiming at harvesting intracorporeal energy to provide continuous power to implanted medical devices. The advantage would be to avoid costly and tedious replacement or recharging of these medical implants. For example, Pfenniger et al. $[12,13]$ propose a prototype that converts the expansion and contraction of an artery (due to pulsatile blood pressure changes) into electrical energy by electromagnetic induction. In their concept, a flexible conductive coil is wound around the artery and an external magnetic field is applied in parallel to the artery's axis. During the systolic phase, the coil expands due to the deformation of the artery, thus inducing an electric field. Another group [14] proposed a "liquid metal magnetohydrodynamic electricity generator" (LMMG). This duct 
system containing liquid metal is placed inside a magnet and the whole device would be attached to certain parts of the human body, such as arm, hand, shoulder, etc. When the electricity conductive liquid metal moves through the magnetic field, an electric potential is formed in the transverse direction and the current can be extracted by an electric load.

\section{iv) Evaluation of tissue engineered constructs}

Tissue-engineered scaffolds are made of biocompatible polymers with various structures that allow one to achieve cell seeding, growth and differentiation. Non-invasive imaging methods are needed to study tissue-engineered constructs before and after implantation. High-resolution MRI combined with efficient intracellular contrast agents shows promise for noninvasive $3 \mathrm{D}$ visualization of tissueengineered constructs before and after in vivo implantation $[15,16]$. Optimizing seeding efficiency, reducing delayed culture periods and mimicking native tissue architecture are crucial requirements for the development of seeding procedures in tissue engineering. In vascular applications, the tubular geometry of the grafts further hampers the efficient delivery of cells onto the scaffold. To overcome these limitations, novel technologies based upon the use of magnetic fields can be used [17,18]. Tubular structures can even be constructed using magnetic force [19].

\section{v) Magnetic drug targeting}

Moreover, there is nowadays focusing attention on applications of magnetic fields acting on magnetic microas well nano-particles carrying target cells or molecules in the research field of magnetic cell and molecule separation, drug targeting, tumor embolization and gene therapy. Magnetic drug targeting by magnetic carriers is a very effective method of forcing drugs to the disease location $[20,21]$. This technique is based on the possibility of encapsulation of target molecules to magnetic micro/nanosphere, or their conjugation on the surface of this magnetic particle. Efficacy of accumulation of carriers depends on several physical and physiological factors, such as the size and type of magnetic particles, their surface character, the gradient and intensity of the applied magnetic field, the distance between the magnetic field source and the desired location of application, the blood flow rate and other hydrodynamic conditions. This magnetic drug targeting helps, for example, to reduce side effects of anticancer agents in surrounding tissue or whole body, due to their enhanced accumulation in magnetically localized volume of tumor $[22,23]$.

Besides, this calculation may also have non-biomedical applications, for example, in industrial situations. And of course, an analytical solution is always useful to test computational codes.

The motion of a conducting fluid through a magnetic field induces an electric field and a potential difference. The resulting current and the applied magnetic field, in turn, produce a Lorentz force on the fluid known as a ponderomotive force. For a Newtonian incompressible fluid, the MHD equations are thus defined by a coupling of Maxwell's electromagnetic equations and Ohm's law, on the one hand, and the Navier-Stokes equations including the Lorentz force on the other hand. An optimal modelisation of the magnetohydrodynamic flow of blood should include the pulsatility of flow, the deformability and conductivity of the vessel wall, together with the induced electrostatic and electromagnetic fields. This leads to a complex mathematical problem and analytical solutions may be found only under restrictive hypotheses.

To our knowledge, the solutions that may be found in the literature assume that the vessel is rigid. Setting a constant pressure gradient, Gold [24] derived expressions for the velocity profile as well as for the induced magnetic field, in the case of a circular tube with non conducting walls placed in a transverse magnetic field. Vardanyan [25] subsequently published an approximate steady solution where the velocity profile and flow rate were calculated by neglecting the induced fields. More recent studies have been essentially based on these founding works, such as the study of Keltner et al. [26], where a comparison was established between the results of Gold and Vardanyan to assess the consequences of neglecting the inductions. With the same hypothesis as Vardanyan, Sud et al. [27] later dealt with a sinusoidal pressure gradient to describe the pulsed nature of blood flow in arteries, and recently, Abi-Abdallah et al. [28] proposed a more realistic arterial flow solution using a physiological pressure gradient model.

The hypothesis of conducting walls was first introduced by Kinouchi et al. [29] who included inductions in the vessel and the surrounding tissues, in the steady flow case, to evaluate the ECG superimposed voltages, using finite elements (numerical solution). Later, Martin et al. [30] reproduced the experimental MHD effect occurring in the aorta through computer simulations on a realistic anatomy, and with a three compartment model: inductionless MHD equations in the aorta (considered as a rigid vessel, with conductive walls), bi-domain equations in the heart, and electrical diffusion in the rest of the body. These compartments are strongly coupled and solved using finite elements.

Other numerical solutions developed in the case of rigid tubes can be found in the literature. For example, Shit [31] proposed an investigation of the flow entrance length behind singularities (for example, near the origin of arterial branches, arterial constrictions and aortic arch) under magnetic environment. He assumed non-conducting walls, and, the induced magnetic and electric fields are not neglected. His code has been tested using the theoretical results that are presented in Abi-Abdallah et al. [32]. Using the Finite Difference Method, Sankar et al. [33] analyzed the pulsatile flow of blood through stenosed arteries in the presence of magnetic field. Due to the presence of the stenosis, they consider the vessel wall as rigid, and, for low Reynolds numbers, they neglect the radial velocity component. They do not deal with induced fields and voltages.

The present work provides an analytical solution including the vessel wall deformability by a coupling of 
A. Drochon: Sinusoïdal flow of blood in a cylindrical deformable vessel exposed to an external magnetic field

equations for the fluid and for the motion of the wall, in the case of simple sinusoidal flow, non-conducting wall and neglected inductions. In other words, the well-known calculation of Womersley [34] is revisited through the inclusion of the Lorentz force in the Navier-Stokes equations.

\section{Position of the problem}

In our problem, the flow is governed by a coupling of:

i) Maxwell's quasi-static electromagnetic equations:

$$
\begin{gathered}
\vec{\nabla} \cdot \vec{E}=\frac{\rho_{e}}{\varepsilon}, \\
\vec{\nabla} \wedge \vec{E}=-\frac{\partial \vec{B}}{\partial t}, \\
\vec{\nabla} \cdot \vec{B}=0, \\
\vec{\nabla} \wedge \vec{B}=\mu \vec{j},
\end{gathered}
$$

ii) Ohm's law:

$$
\vec{j}=\sigma(\vec{E}+\vec{u} \wedge \vec{B})
$$

iii) Navier-Stokes equations:

$$
\rho\left(\frac{\partial \vec{u}}{\partial t}+(\vec{u} \cdot \vec{\nabla}) \vec{u}\right)=-\vec{\nabla} P+\eta \Delta \vec{u}+\vec{j} \wedge \vec{B},
$$

iv) fluid conservation equation (incompressible fluid):

$$
\vec{\nabla} \cdot \vec{u}=0
$$

where $\vec{u}$ and $P$ represent the fluid velocity $(\mathrm{m} / \mathrm{s})$ and pressure $(\mathrm{Pa}), \vec{E}$ and $\vec{B}$ are the electric $(\mathrm{V} / \mathrm{m})$ and magnetic (T) fields, $\vec{j}$ the electric current density $\left(\mathrm{A} / \mathrm{m}^{2}\right), \varepsilon$ the electric permittivity $(\mathrm{F} / \mathrm{m}), \rho_{e}$ the charge density $\left(\mathrm{C} / \mathrm{m}^{3}\right), \mu$ the magnetic permeability $(\mathrm{H} / \mathrm{m}) ; \eta, \sigma, \rho$ represent respectively the fluid viscosity $(\mathrm{Pa} \mathrm{s})$, conductivity $(\mathrm{S} / \mathrm{m})$ and density $\left(\mathrm{kg} / \mathrm{m}^{3}\right)$.

If the flow configuration is defined as in Figure 1, the components of the magnetic field vector in the cylindrical frame are:

$$
\vec{B}=\left(B_{0} \cos \theta,-B_{0} \sin \theta, B_{I}(t, r, \theta)\right),
$$

with $B_{I} \ll B_{0}$. The induced magnetic field $B_{I}$ does not depend on $z$; this is consistent with equation (3).

\subsection{Induced fields neglected}

Some authors $[25,27]$ have suggested that when the magnetic Reynolds number, $\operatorname{Re}_{m},\left(\operatorname{Re}_{m}=\mathrm{Ru}_{0} \sigma \mu\right.$, with $u_{0}$ a characteristic velocity of the flow) is small, the induced fields $E_{I}$ and $B_{I}$ can be neglected.

In that case, it comes from (5) that: $j=\sigma(u \wedge B)$. Abi-Abdallah et al. $[32,35]$ have shown that this approximation is acceptable for low $B_{0}$ fields, such as those used in biomedical applications.

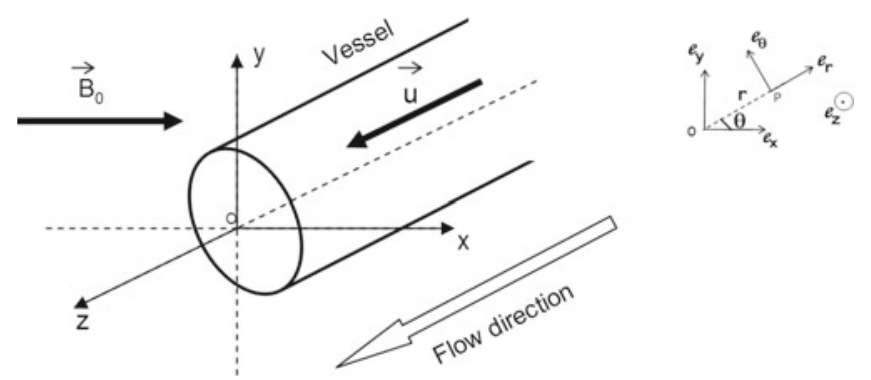

Fig. 1. Flow model geometry. The vessel is represented by a cylindrical conduct where blood flows along the $O z$ axis, in the presence of a transverse steady magnetic field oriented in the $O x$ direction.

\subsection{Fluid motion}

The problem is solved in the plane $\theta=\pi / 2$, where the Hall effect is maximal. Some artificial axisymmetry can then be obtained by a rotation of this plane around the $z$-axis. This solution thus provides an overestimation of the studied effet.

The velocity and magnetic fields are:

$$
\vec{u}=\left(u_{r}, 0, u_{z}\right) \text { and } \vec{B}=\left(0,-B_{0}, 0\right) .
$$

The corresponding Lorentz force is:

$$
\vec{j} \wedge \vec{B}=[\sigma(\vec{u} \wedge \vec{B})] \wedge \vec{B}=-\sigma B_{0}^{2} \mid \begin{aligned}
& u_{r} \\
& 0 \\
& u_{z}
\end{aligned}
$$

The radial and axial projections of Navier-Stokes equation (6) are:

$$
\begin{aligned}
\frac{\partial u_{r}}{\partial t}= & -\frac{1}{\rho}\left(\frac{\partial P}{\partial r}\right)+\nu\left(\frac{\partial^{2} u_{r}}{\partial r^{2}}+\frac{1}{r} \frac{\partial u_{r}}{\partial r}+\frac{\partial^{2} u_{r}}{\partial z^{2}}-\frac{u_{r}}{r^{2}}\right) \\
& -\frac{\sigma u_{r} B_{0}^{2}}{\rho}
\end{aligned}
$$

$\frac{\partial u_{z}}{\partial t}=-\frac{1}{\rho}\left(\frac{\partial P}{\partial z}\right)+\nu\left(\frac{\partial^{2} u_{z}}{\partial r^{2}}+\frac{1}{r} \frac{\partial u_{z}}{\partial r}+\frac{\partial^{2} u_{z}}{\partial z^{2}}\right)-\frac{\sigma u_{z} B_{0}^{2}}{\rho}$

where $\nu$ is the fluid kinematic viscosity.

The amplitude of the pressure disturbance is sufficiently small for the nonlinear terms of the inertia of the fluid to be considered negligible in comparison with the linear ones [36,37].

The continuity equation (7) yields:

$$
\frac{\partial u_{r}}{\partial r}+\frac{u_{r}}{r}+\frac{\partial u_{z}}{\partial z}=0
$$

Another equation may be added, taking the divergence of equation (6) (with convective acceleration terms neglected). One obtains, using equations (10) and (13):

$$
\Delta P=\operatorname{div}(j \wedge B)=0 .
$$


The equations to solve for the fluid motion are thus (11)-(14).

We seek solutions of this system of equations for the case of the propagation of forced pressure waves which are harmonic in $t$ and in $z$. We will assume that $u_{r}, u_{z}$ and $P$ vary in the following manner:

$$
\begin{aligned}
P(r, t, z) & =P^{*}(r) \exp \left[i \omega\left(t-\frac{z}{c}\right)\right], \\
u_{r}(r, t, z) & =u_{r}^{*}(r) \exp \left[i \omega\left(t-\frac{z}{c}\right)\right], \\
u_{z}(r, t, z) & =u_{z}^{*}(r) \exp \left[i \omega\left(t-\frac{z}{c}\right)\right],
\end{aligned}
$$

where $\omega$ denotes the circular frequency of the forced oscillation and $c$ denotes the velocity of propagation of the oscillation.

The solutions may be expressed as:

$$
\begin{gathered}
P^{*}(r)=\frac{\eta c}{\omega} \frac{\alpha^{\prime 2}}{R^{2}} C_{1} J_{0}\left(i \frac{\omega}{c} r\right), \\
u_{z}^{*}(r)=C_{1} J_{0}\left(i \frac{\omega}{c} r\right)+C_{2} \frac{J_{0}\left(\delta^{\prime} \frac{r}{R} i^{3 / 2}\right)}{J_{0}\left(\delta^{\prime} i^{3 / 2}\right)}, \\
u_{r}^{*}(r)=C_{1} J_{1}\left(i \frac{\omega}{c} r\right)+\frac{R i \omega C_{2}}{c \delta^{\prime} i^{3 / 2}} \frac{J_{1}\left(\delta^{\prime} \frac{r}{R} i^{3 / 2}\right)}{J_{0}\left(i^{3 / 2} \delta^{\prime}\right)},
\end{gathered}
$$

where $C_{1}$ and $C_{2}$ are integration constants $(\mathrm{m} / \mathrm{s}), J_{0}(x)$ and $J_{1}(x)$ denote the first kind zeroth and first order Bessel functions, and the dimensionless parameters $\alpha^{\prime}$ and $\delta^{\prime}$ are given by the following relations:

$$
\alpha^{\prime}=R \sqrt{\frac{\omega}{\nu}-i \frac{M^{2}}{R^{2}}} \quad \text { and } \quad \delta^{\prime}=\sqrt{\alpha^{\prime 2}-i \frac{\omega^{2}}{c^{2}} R^{2}} .
$$

The Hartmann number, $M$, represents the ratio of the magnetic forces to the viscous forces and is defined as:

$$
M=R B_{0} \sqrt{\frac{\sigma}{\eta}} .
$$

We notice that when $M=0$ (no magnetic field), the parameter $\alpha^{\prime}$ reduces to the classical Womersley number.

\subsection{Vessel wall motion}

To express the equilibrium of a surface element of the vessel wall, we follow the analysis of Atabek and Lew [37] and Flaud and Rogova [38].

Let $\zeta_{r}(z, t)$ and $\zeta_{z}(z, t)$ denote the radial and longitudinal displacement of a point of the wall due to the wave motion, $h$ the thickness of the wall $(h \ll R), \sigma_{p}$ and $E$ its Poisson's ratio and Young modulus, and $\rho_{w}$, its density. The strains in the longitudinal direction and in the circumferential direction are thus: $e_{L}=\frac{\partial \zeta_{z}}{\partial z}$ and $e_{c}=\frac{\zeta_{r}}{R}$.

Using these notations, the equations of motion of the wall may be written as:
Radial projection:

$$
\begin{aligned}
\rho_{w} h \frac{\partial^{2} \zeta_{r}}{\partial t^{2}}= & {\left[P-2 \eta \frac{\partial u_{r}}{\partial r}\right]_{r=R}-\frac{E h}{R\left(1-\sigma_{p}^{2}\right)}\left(\frac{\zeta_{r}}{R}+\sigma_{p} \frac{\partial \zeta_{z}}{\partial z}\right) } \\
& +\frac{\zeta_{r}}{R_{0}^{2}} T_{\theta 0}+T_{t 0} \frac{\partial^{2} \zeta_{r}}{\partial z^{2}}
\end{aligned}
$$

Longitudinal projection:

$$
\begin{aligned}
\rho_{w} h \frac{\partial^{2} \zeta_{z}}{\partial t^{2}}= & -\eta\left[\frac{\partial u_{z}}{\partial r}+\frac{\partial u_{r}}{\partial z}\right]_{r=R}+\frac{E h}{\left(1-\sigma_{p}^{2}\right)} \\
& \times\left(\frac{\partial^{2} \zeta_{z}}{\partial z^{2}}+\frac{\sigma_{p}}{R} \frac{\partial \zeta_{r}}{\partial z}\right)+\left(\frac{\partial \zeta_{r}}{\partial z}\right)\left(\frac{T_{t 0}-T_{\theta 0}}{R}\right),
\end{aligned}
$$

where the left hand side of the equations represent the inertia terms, the terms $\left[P-2 \eta \frac{\partial u_{r}}{\partial r}\right]_{r=R}$ and $-\eta$ $\left[\frac{\partial u_{z}}{\partial r}+\frac{\partial u_{r}}{\partial z}\right]_{r=R}$ are the surface forces exerted by the fluid on the vessel wall, and the stress-strain relations in the isotropic Hookean wall are expressed as:

$$
\begin{aligned}
& T_{\theta}-T_{\theta 0}=\frac{E h}{\left(1-\sigma_{p}^{2}\right)}\left(\frac{\zeta_{r}}{R}+\sigma_{p} \frac{\partial \zeta_{z}}{\partial z}\right), \\
& T_{t}-T_{t 0}=\frac{E h}{\left(1-\sigma_{p}^{2}\right)}\left(\frac{\partial \zeta_{z}}{\partial z}+\sigma_{p} \frac{\zeta_{r}}{R}\right),
\end{aligned}
$$

where $T_{\theta}$ denotes the circumferential stress and $T_{t}$ the tangential stress $\left(T_{\theta 0}\right.$ and $T_{t 0}$ are the initial stresses, before the wave moves along the tube). Tensile stresses can be assumed as uniformly distributed across the thickness of the tube.

\subsection{Boundary conditions}

The velocity of the fluid particles on the wall must be equal to the velocity of the wall:

$$
u_{r}=\frac{\partial \zeta_{r}}{\partial t} \text { and } u_{z}=\frac{\partial \zeta_{z}}{\partial t}, \text { for } r=R
$$

The displacements which correspond to the forced harmonic oscillations described in equation (15) will have the following form:

$$
\begin{aligned}
& \zeta_{r}(t, z)=C_{3} \exp \left[i \omega\left(t-\frac{z}{c}\right)\right], \\
& \zeta_{z}(t, z)=C_{4} \exp \left[i \omega\left(t-\frac{z}{c}\right)\right] .
\end{aligned}
$$

\section{Solution of the problem}

The constants $C_{1}, C_{2}, C_{3}, C_{4}$ will be determined by solving the system of four equations obtained using (21), (22) and (25), with the fluid velocity given by (15), (17) and (18) and the wall displacements given by (26) 
A. Drochon: Sinusoïdal flow of blood in a cylindrical deformable vessel exposed to an external magnetic field

This system can be written as:

$$
\left[\begin{array}{llll}
a_{11} & a_{12} & a_{13} & a_{14} \\
a_{21} & a_{22} & a_{23} & a_{24} \\
a_{31} & a_{32} & a_{33} & a_{34} \\
a_{41} & a_{42} & a_{43} & a_{44}
\end{array}\right]\left[\begin{array}{l}
C_{1} \\
C_{2} \\
C_{3} \\
C_{4}
\end{array}\right]=\left[\begin{array}{l}
0 \\
0 \\
0 \\
0
\end{array}\right]
$$

where the $\left[a_{i j}\right]$ factors are given by:

$$
\left[\begin{array}{cccc}
J_{1}\left(i \frac{\omega}{c} R\right) & \frac{R \omega}{c \delta^{\prime} \sqrt{i}} \frac{J_{1}\left(\delta^{\prime} i^{3 / 2}\right)}{J_{0}\left(\delta^{\prime} i^{3 / 2}\right)} & -i \omega & 0 \\
J_{0}\left(i \frac{\omega}{c} R\right) & 1 & 0 & -i \omega \\
a_{31} & a_{32} & a_{33} & \frac{E h \sigma_{p} i \omega}{R c\left(1-\sigma_{p}^{2}\right)} \\
2 \frac{\eta \omega i}{c} J_{1}\left(i \frac{\omega}{c} R\right) & a_{42} & a_{43} & \rho_{w} h \omega^{2}-\frac{E h \omega^{2}}{\left(1-\sigma_{p}^{2}\right) c^{2}}
\end{array}\right]
$$

and

$$
\begin{aligned}
a_{31}= & \frac{\eta c}{\omega} \frac{\alpha^{\prime 2}}{R^{2}} J_{0}\left(i \frac{\omega}{c} R\right)-2 \eta \frac{i \omega}{c} J_{0}\left(i \frac{\omega}{c} R\right) \\
& +2 \eta \frac{1}{R} J_{1}\left(i \frac{\omega}{c} R\right), \\
a_{32}= & -\frac{2 \eta i \omega}{c}\left[1-\frac{1}{\delta^{\prime} i^{3 / 2}} \frac{J_{1}\left(\delta^{\prime} i^{3 / 2}\right)}{J_{0}\left(\delta^{\prime} i^{3 / 2}\right)}\right], \\
a_{33}= & \rho_{w} h \omega^{2}-\frac{E h}{R^{2}\left(1-\sigma_{p}^{2}\right)}+\frac{T_{\theta 0}}{R_{0}^{2}}-\frac{\omega^{2}}{c^{2}} T_{t 0}, \\
a_{42}= & \eta\left[\frac{\delta^{\prime} i^{3 / 2}}{R}-\frac{R \omega^{2}}{c^{2} \delta^{\prime} i^{3 / 2}}\right] \frac{J_{1}\left(\delta^{\prime} i^{3 / 2}\right)}{J_{0}\left(\delta^{\prime} i^{3 / 2}\right)}, \\
a_{43}= & -\frac{i \omega}{R c}\left[\frac{E h \sigma_{p}}{\left(1-\sigma_{p}^{2}\right)}+T_{t 0}-T_{\theta 0}\right] .
\end{aligned}
$$

This system has a nontrivial solution if the determinant of the coefficients of the system is equal zero. This means that:

$a_{11} \Delta_{11}-a_{12} \Delta_{12}+a_{13} \Delta_{13}=0$, where $\Delta_{i j}$ is the co-factor of the $a_{i j}$ term.

Before expanding the determinant, we introduce additional hypotheses and numerical data (Tab. 1):

- the vessel wall is incompressible, so that its Poisson's ratio, $\sigma_{p}$, is 0.5 ;

- the vessel has no pre-stress: $T_{\theta 0}=T_{t 0}=0$;

- the wavelengths of the oscillations are very large compared with the radius of the tube, meaning that $\omega R / c$ $\ll 1$;

- the velocity of wave propagation in an incompressible, inviscid fluid enclosed in a thin-walled elastic tube, $c_{0}$, is given by the Moens-Korteweg equation: $c_{0}^{2}=\frac{E h}{2 \rho R}$. Using the numerical values listed in Table 1 , this yields $c_{0}=9.76 \mathrm{~m} / \mathrm{s}$. The term $\omega R / c_{0}$ is thus of order $8 \times$ $10^{-3}$ and this justifies the long wavelength hypothesis.
Table 1. Numerical data used in the calculations of this paper.

\begin{tabular}{ccc}
\hline Quantity & Symbol & Value \\
\hline $\begin{array}{c}\text { Radius of the vessel } \\
\text { Cardiac frequency }\end{array}$ & $\omega / 2 \pi$ & $0.01 \mathrm{~m}$ \\
Circular frequency & $\omega$ & $7.854 \mathrm{rd} / \mathrm{s}$ \\
$\begin{array}{c}\text { Young modulus } \\
\text { of aortic wall } \\
\text { Thickness of } \\
\text { the wall }\end{array}$ & $E$ & $10^{6} \mathrm{~Pa}$ \\
$\quad$ Wall density & $\rho_{w}$ & $1100 \mathrm{~kg} / \mathrm{m}^{3}$ \\
$\quad \begin{array}{c}\text { Blood density } \\
\text { Blood dynamic } \\
\text { viscosity }\end{array}$ & $\rho$ & $1050 \mathrm{~kg} / \mathrm{m}^{3}$ \\
$\begin{array}{c}\text { Blood electrical } \\
\text { conductivity }\end{array}$ & $\eta$ & $4 \times 10^{-3} \mathrm{~Pa} \mathrm{~s}$ \\
$\begin{array}{c}\text { Magnetic permeability } \\
\text { Characteristic velocity } \\
\text { of blood in the aorta } \\
\text { Magnetic Reynolds } \\
\text { number }\end{array}$ & $\mu$ & $0.5 \mathrm{~S} / \mathrm{m}$ \\
"Classical" Womersley \\
number
\end{tabular}

Moreover, the Bessel functions may be approximated as:

$$
J_{0}\left(i \frac{\omega}{c} R\right) \approx 1 \quad \text { and } \quad J_{1}\left(i \frac{\omega}{c} R\right)=\frac{i \omega R}{2 c} .
$$

The quantity $\rho_{w} h \omega^{2}$ may also be neglected in comparison to $\frac{4 E h}{3 R^{2}}$.

Introducing a notation similar to that used by Womersley [34],

$$
F_{10}^{\prime}\left(\delta^{\prime}\right)=\frac{2 J_{1}\left(\delta^{\prime} i^{3 / 2}\right)}{\delta^{\prime} i^{3 / 2} J_{0}\left(\delta^{\prime} i^{3 / 2}\right)},
$$

the matrix (28) becomes

$$
\text { see equation (31) at the bottom of this page. }
$$

After some algebraic manipulations on the rows and the columns of the determinant (following the method described in Atabek and Lew [37]), we obtain the so-called frequency equation (32), which unknown is the ratio $\left(c_{0} / c\right)$.

$$
\begin{aligned}
\frac{16}{3}(1 & \left.-F_{10}^{\prime}\left(\delta^{\prime}\right)\right)\left(\frac{c_{0}^{4}}{c^{4}}\right)+\left(\frac{c_{0}^{2}}{c^{2}}\right)\left[-\frac{8}{3}\left(1-F_{10}^{\prime}\left(\delta^{\prime}\right)\right) k\right. \\
& \left.+\frac{4}{3} K\left(F_{10}^{\prime}\left(\delta^{\prime}\right)-4\right)\right]+2 K k+\frac{\delta^{\prime 2}}{R^{2}} \frac{K \nu}{\omega} F_{10}^{\prime}\left(\delta^{\prime}\right)=0
\end{aligned}
$$

$$
\left[\begin{array}{cccc}
\frac{i \omega R}{2 c} & \frac{R \omega i}{2 c} F_{10}^{\prime}\left(\delta^{\prime}\right) & -i \omega & 0 \\
1 & 1 & 0 & -i \omega \\
\rho c-\frac{\eta c}{\omega} i \frac{M^{2}}{R^{2}}-\eta i \frac{\omega}{c} & -\frac{\eta i \omega}{c}\left(2-F_{10}^{\prime}\left(\delta^{\prime}\right)\right) & -\frac{4}{3} \frac{E h}{R^{2}} & \frac{2}{3} \frac{E h i \omega}{R c} \\
-\frac{\eta \omega^{2}}{c^{2}} R & \eta\left[\frac{\delta^{\prime 2} i^{3}}{R^{2}}-\frac{\omega^{2}}{c^{2}}\right] \frac{R}{2} F_{10}^{\prime}\left(\delta^{\prime}\right) & -\frac{2}{3} E h \frac{i \omega}{R c} & \rho_{w} h \omega^{2}-\frac{4 E h \omega^{2}}{3 c^{2}}
\end{array}\right]
$$


The European Physical Journal Applied Physics

Table 2. Solutions of the frequency equation (32): non-dimensional celerities for the two types of waves (Young mode, $c_{1} / c_{0}$, and Lamb mode, $c_{2} / c_{0}$ ), obtained for selected values of the Hartmann number $M$. The moduli of the celerities $c_{1}$ and $c_{2}$ are also given.

\begin{tabular}{ccccccc}
\hline$M$ & 0 & 1.2 & 2 & 4 & 10 & 20 \\
\hline$c_{1} / c_{0}$ & $0.9611+0.0281 \mathrm{i}$ & $0.9609+0.0313 \mathrm{i}$ & $0.9605+0.0369 \mathrm{i}$ & $0.9571+0.0632 \mathrm{i}$ & $0.8836+0.2188 \mathrm{i}$ & $0.5604+0.3434 \mathrm{i}$ \\
$c_{2} / c_{0}$ & $3.1329+0.3523 \mathrm{i}$ & $3.1333+0.3535 \mathrm{i}$ & $3.1340+0.3558 \mathrm{i}$ & $3.1371+0.3663 \mathrm{i}$ & $3.1493+0.4381 \mathrm{i}$ & $3.1201+0.6552 \mathrm{i}$ \\
$\left|c_{1}\right|(\mathrm{m} / \mathrm{s})$ & 9.3843 & 9.3833 & 9.3814 & 9.3616 & 8.8844 & 6.4147 \\
$\left|c_{2}\right|(\mathrm{m} / \mathrm{s})$ & 30.7698 & 30.775 & 30.7843 & 30.8261 & 31.0331 & 31.1164 \\
\hline
\end{tabular}

Table 3. Correspondence between the $B_{0}$ fields and the Hartmann numbers, using the numerical data of Table 1.

\begin{tabular}{ccccccc}
\hline$M$ & 0 & 1.2 & 2 & 4 & 10 & 20 \\
\hline$B_{0}(\mathrm{~T})$ & 0 & 10.73 & 17.89 & 35.78 & 89.44 & 178.88 \\
\hline
\end{tabular}

In equation (32), two non-dimensional coefficients, $k$ and $K$, have been introduced. They are defined as follows:

$$
\begin{aligned}
k & =\frac{\rho_{w} h}{\rho R}, \text { and } K=\left(\frac{\alpha_{\text {magnetic }}}{\alpha_{\text {Womersley }}}\right)^{2}=\frac{\alpha^{\prime 2}}{\alpha^{2}} \\
& =\frac{R^{2} \frac{\omega}{\nu}\left(1-i \frac{M^{2}}{R^{2}} \frac{\nu}{\omega}\right)}{R^{2} \frac{\omega}{\nu}}=1-i \frac{M^{2}}{R^{2}} \frac{\nu}{\omega} .
\end{aligned}
$$

In the case where $M=0$ (no magnetic field), the parameter $K=1$, and equation (32) reduces to the frequency equation given by Atabek and Lew [37] (their Eq. (59)).

Equation (32) may be first considered as quadratic in $\left(c_{0} / c\right)^{2}$, and then the square root of $\left(c_{0} / c\right)^{2}$ is taken. Consequently, two of the solutions of equation (32) differ from the other two only in sign, representing outgoing waves traveling in the axial direction and the corresponding incoming waves. Following Atabek and Lew [37], we consider only the two outgoing waves, and denote by $c_{1}$ and $c_{2}$ their propagation velocities. The waves having the smaller propagation velocity are generally referred to as the waves of the first kind (Young mode, representing pressures waves propagating in the fluid and associated with radial displacement of the wall), while the other type of waves are called the waves of the second kind (Lamb mode, representing waves propagating in the vessel wall under blood loading, associated with longitudinal displacement of the wall). It is mainly the Young mode that is palpable at wrist or neck arteries [39]. The Lamb mode is neglected in most of the physiological situations, because the vessel walls are tethered to the surrounding tissues, and their longitudinal movements are thus limited.

The roots of equation (32) for the two types of waves are given in Table 2, for selected values of the Hartmann number $M$. These celerities will be commented in Section 4. The correspondence between Hartmann numbers and $B_{0}$ field values can be obtained using the data of Table 1, and is presented in Table 3 .

After determining the celerities $c$, it is necessary to go back to the system of equations written in the form (27)(28), and try to solve it for the constants $C_{1}, C_{2}, C_{3}$, $C_{4}$. However, since the determinant of the matrix given in equations (27) and (28) equals zero, only three of this four constants are independent (the matrix is reduced to order 3$)$. The constant $C_{1}$ is choosen as the free parameter because it is directly related to the amplitude of the pressure perturbation (Eq. (16)). The system to solve is now:

$$
\begin{gathered}
C_{1}\left[\frac{i \omega R}{2 c}+\frac{i \omega R}{2 c} F_{10}^{\prime}\left(\delta^{\prime}\right) \frac{C_{2}}{C_{1}}-i \omega \frac{C_{3}}{C_{1}}\right]=0, \\
C_{1}\left[1+\frac{C_{2}}{C_{1}}-i \omega \frac{C_{4}}{C_{1}}\right]=0, \\
C_{1}\left[\rho c-\eta i\left(\frac{\omega}{c}+\frac{c}{\omega} \frac{M^{2}}{R^{2}}\right)+\frac{\eta i \omega}{c}\left(F_{10}^{\prime}\left(\delta^{\prime}\right)-2\right) \frac{C_{2}}{C_{1}}\right. \\
\left.-\frac{4}{3} \frac{E h}{R^{2}} \frac{C_{3}}{C_{1}}+\frac{2}{3} \frac{E h i \omega}{R c} \frac{C_{4}}{C_{1}}\right]=0 .
\end{gathered}
$$

Solving by substitution, we obtain:

$$
\begin{gathered}
\frac{C_{2}}{C_{1}}=-\frac{K-\frac{\omega \nu i}{c^{2}}}{\left[-\frac{\nu i \omega}{c^{2}}\left(2-F_{10}^{\prime}\left(\delta^{\prime}\right)\right)+\frac{2}{3} \frac{E h}{R \rho c^{2}}\left(1-F_{10}^{\prime}\left(\delta^{\prime}\right)\right)\right]} \\
\frac{C_{3}}{C_{1}}=\frac{R}{2 c}+\frac{R}{2 c} F_{10}^{\prime}\left(\delta^{\prime}\right) \frac{C_{2}}{C_{1}} \\
\frac{C_{4}}{C_{1}}=\frac{1}{i \omega}\left(1+\frac{C_{2}}{C_{1}}\right) .
\end{gathered}
$$

Finally, substituting equation (37) in (17) and (18), one obtains the non-dimensional fluid velocities:

$$
\begin{gathered}
\frac{u_{z}^{*}(r)}{C_{1}}=1+\frac{C_{2}}{C_{1}} \frac{J_{0}\left(\delta^{\prime} \frac{r}{R} i^{3 / 2}\right)}{J_{0}\left(\delta^{\prime} i^{3 / 2}\right)}, \quad \text { because } \quad J_{0}\left(\frac{i \omega r}{c}\right) \approx 1, \\
\frac{u_{r}^{*}(r)}{C_{1}}=\frac{i \omega R}{c}\left[\frac{r}{2 R}+\frac{C_{2}}{C_{1}} \frac{1}{\delta^{\prime} i^{3 / 2}} \frac{J_{1}\left(\delta^{\prime} \frac{r}{R} i^{3 / 2}\right)}{J_{0}\left(\delta^{\prime} i^{3 / 2}\right)}\right], \\
\text { because } \quad J_{1}\left(\frac{i \omega r}{c}\right) \approx \frac{i \omega r}{2 c} .
\end{gathered}
$$

Similarly, substituting equations (38) and (39) in equation (26), the wall displacements can be expressed in terms of $C_{1}$ :

$$
\begin{aligned}
\frac{\zeta_{r}(t, z)}{C_{1}} & =\frac{C_{3}}{C_{1}} \exp \left[i \omega\left(t-\frac{z}{c}\right)\right] \\
\frac{\zeta_{z}(t, z)}{C_{1}} & =\frac{C_{4}}{C_{1}} \exp \left[i \omega\left(t-\frac{z}{c}\right)\right] .
\end{aligned}
$$


A. Drochon: Sinusoïdal flow of blood in a cylindrical deformable vessel exposed to an external magnetic field
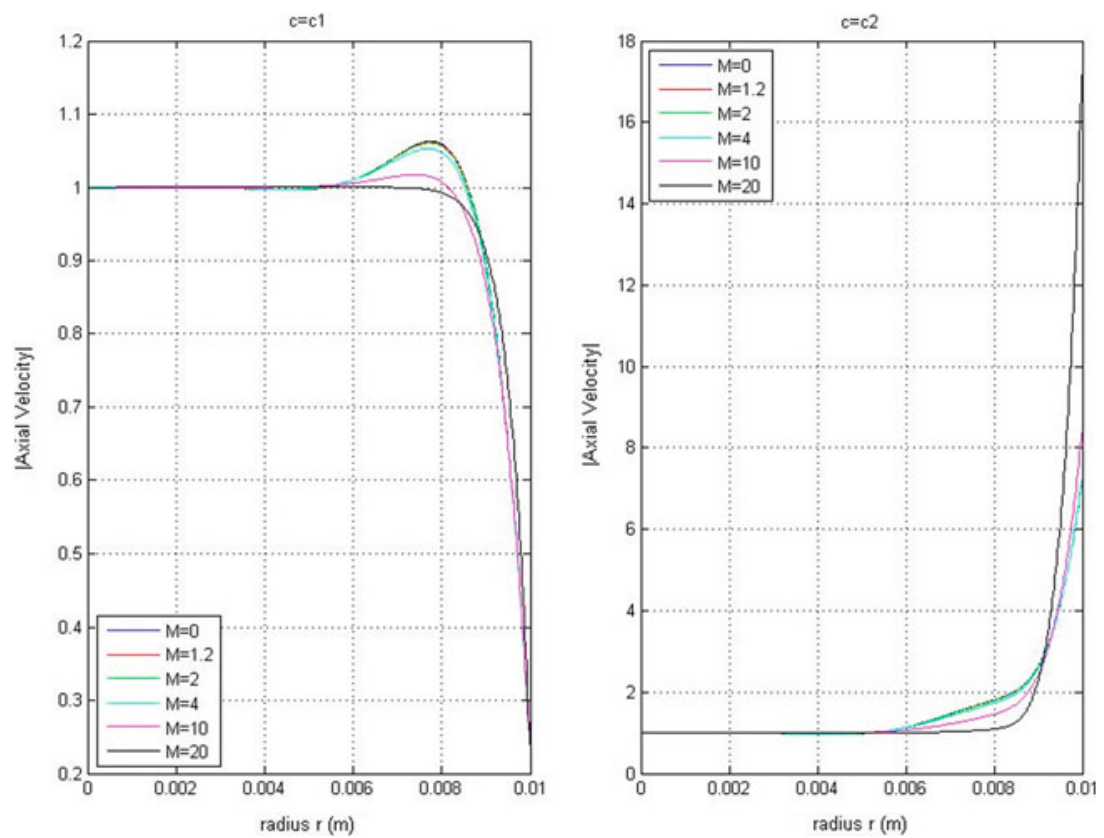

Fig. 2. Moduli of the amplitude of the non-dimensional axial velocity, $\left|\frac{u_{z}^{*}(r)}{C_{1}}\right|$, for the two types of waves (Young waves, $c=c_{1}$, and Lamb waves, $c=c_{2}$ ), and for different values of the Hartmann number $M$.

Using equations (40) and (41), it is easy to verify that the velocities at the wall are the derivatives of the corresponding displacements (calculated from Eq. (42)) with respect to time:

$$
u_{z}(R)=i \omega \zeta_{z}, \text { and } u_{r}(R)=i \omega \zeta_{r}
$$

This is of course consistent with the boundary condition (25).

Besides, the mean axial velocity (amplitude) may be calculated as follows:

$$
\overline{u_{z}^{*}(r)}=\frac{1}{\pi R^{2}} \int_{0}^{R} 2 \pi r \times u_{z}^{*}(r) \mathrm{d} r .
$$

After integration of equation (40), we obtain:

$$
\frac{\overline{u_{z}^{*}(r)}}{C_{1}}=1+\frac{C_{2}}{C_{1}} F_{10}^{\prime}\left(\delta^{\prime}\right) .
$$

Looking at equation (38), it appears that the amplitude of the radial displacements is directly related to the flow rate in the vessel, since we have:

$$
\frac{C_{3}}{C_{1}}=\frac{R}{2 c} \frac{\overline{u_{z}^{*}(r)}}{C_{1}} .
$$

A similar relation had been previously obtained by Womersley [34]. It indicates that the amount of flow will be proportionately greater in that vessel which is more elastic. We also have:

$$
u_{r}^{*}(R)=\frac{i \omega R}{2 c} \overline{u_{z}^{*}(r)} .
$$

Equation (47) shows that the radial velocity at the wall is also proportional to the flow rate.

\section{Results and discussion}

\subsection{Wave celerities}

The wave celerities (roots of Eq. (32)) are presented in Table 2. First of all, it is important to mention that the values obtained in the case $M=0$ are in full agreement with the values previously published in papers who studied the propagation of pressure pulse in elastic vessels without magnetic field $[36,37,40,41]$. As it is the case in any of those papers, we find that the second type of waves (Lamb waves), propagates much faster than the first type of waves (Young waves). The celerity $c_{1}$ obtained in our calculation in the case $M=0(9.38 \mathrm{~m} / \mathrm{s})$ falls in the range of physiological values given by Wang and Parker [42].

In the presence of a magnetic field $(M \neq 0)$, the celerities $c_{1}$ and $c_{2}$ show inverse tendencies: the moduli of $c_{1}$ decrease when $B_{0}$ increases, whereas the moduli of $c_{2}$ increase. However, the impact of the magnetic field on $c_{2}$ is less important than on $c_{1}$ (may be because we have neglected any direct effect of the magnetic field on the wall itself: the magnetic field acts on the fluid, which, in turn, acts on the vessel wall). It is important to note that such an effect becomes significant only for Hartmann numbers higher than $4\left(B_{0} \approx 35 \mathrm{~T}\right)$. This means that any effects are negligible in classical MRI examinations and measurements $\left(B_{0}=1.5\right.$ or $\left.3 \mathrm{~T}\right)$. We can also observe that, even at very high Hartmann number $(M=20)$, the reduction in $c_{1}$ is about $30 \%$. Such a range of values $(6-10 \mathrm{~m} / \mathrm{s})$ corresponds to the variability of the results obtained in experimental measurements of pulse wave velocity with MRI $[8-11,43,44]$. This dispersion would easily mask the influence of the magnetic field, even at high $B_{0}$. 
The European Physical Journal Applied Physics
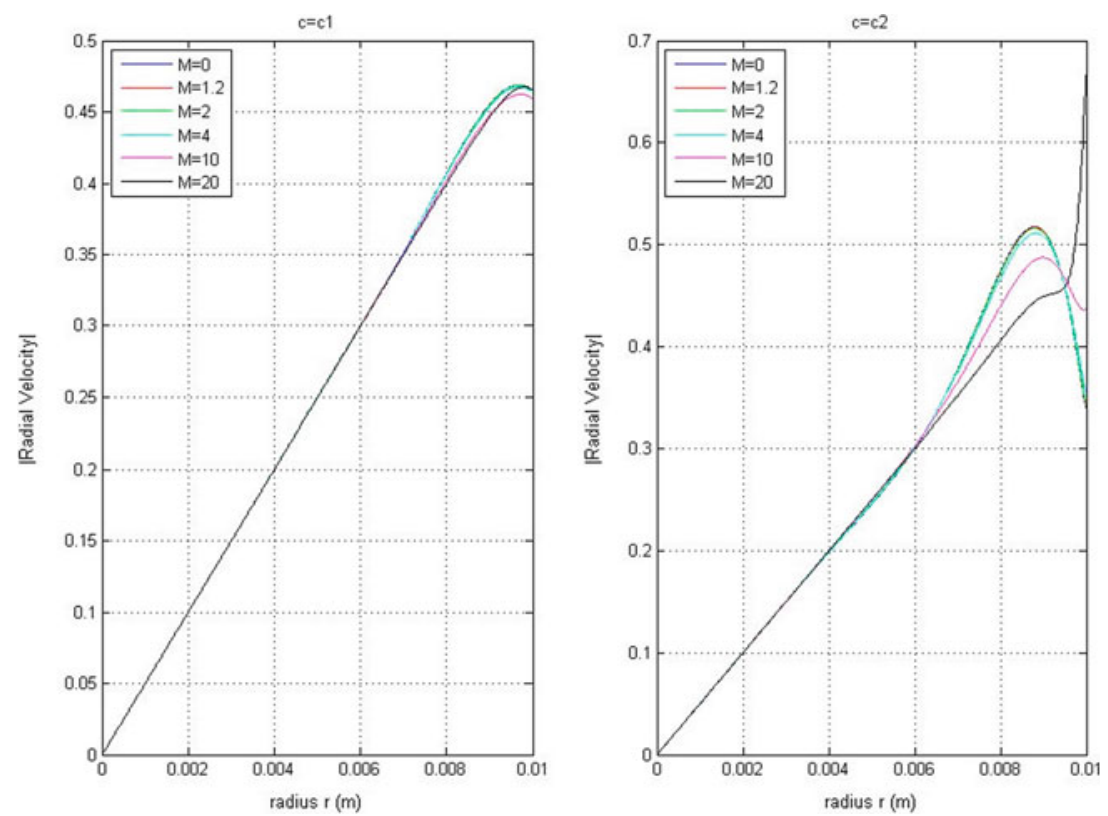

Fig. 3. Moduli of the amplitude of the non-dimensional radial velocity, $\left|\frac{u_{r}^{*}(r)}{C_{1}} \frac{c}{\omega R}\right|$, plotted for the two types of waves (Young waves, $c=c_{1}$, and Lamb waves, $c=c_{2}$ ), and for different values of the Hartmann number.
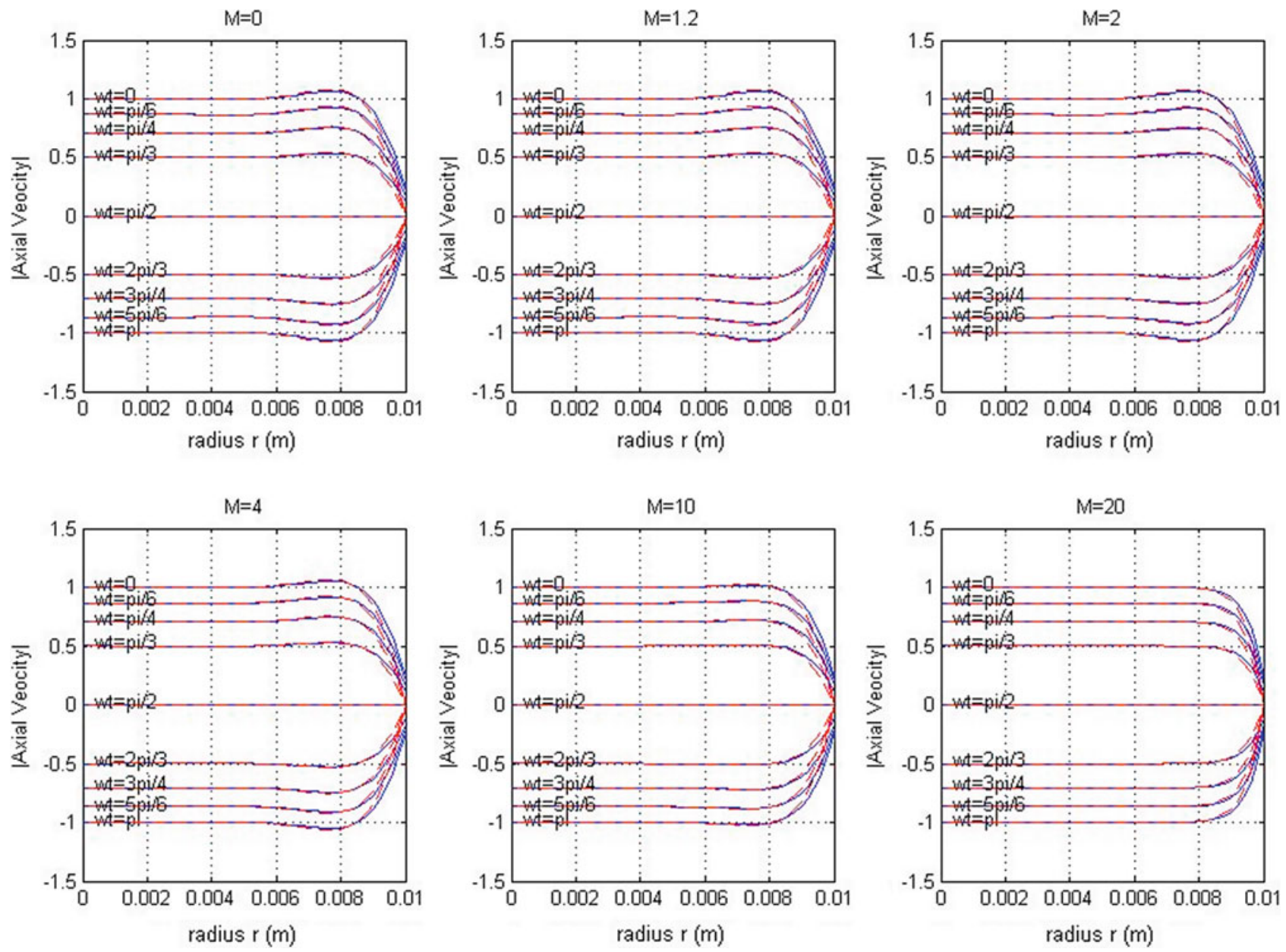

Fig. 4. The quantity $\Re\left(\left|\frac{u_{z}^{*}(r)}{u_{z}^{*}(0)}\right| e^{i \omega t}\right)$ is plotted for the deformable tube (solution of this paper - full lines) and for the rigid tube (solution of Sud et al. [27] - dotted lines), for slow waves (celerity $c_{1}$ ), and for different values of the Hartmann number $M$. For practical reasons, a shortened notation has been used for the ordinates of the graphs. 
A. Drochon: Sinusoïdal flow of blood in a cylindrical deformable vessel exposed to an external magnetic field
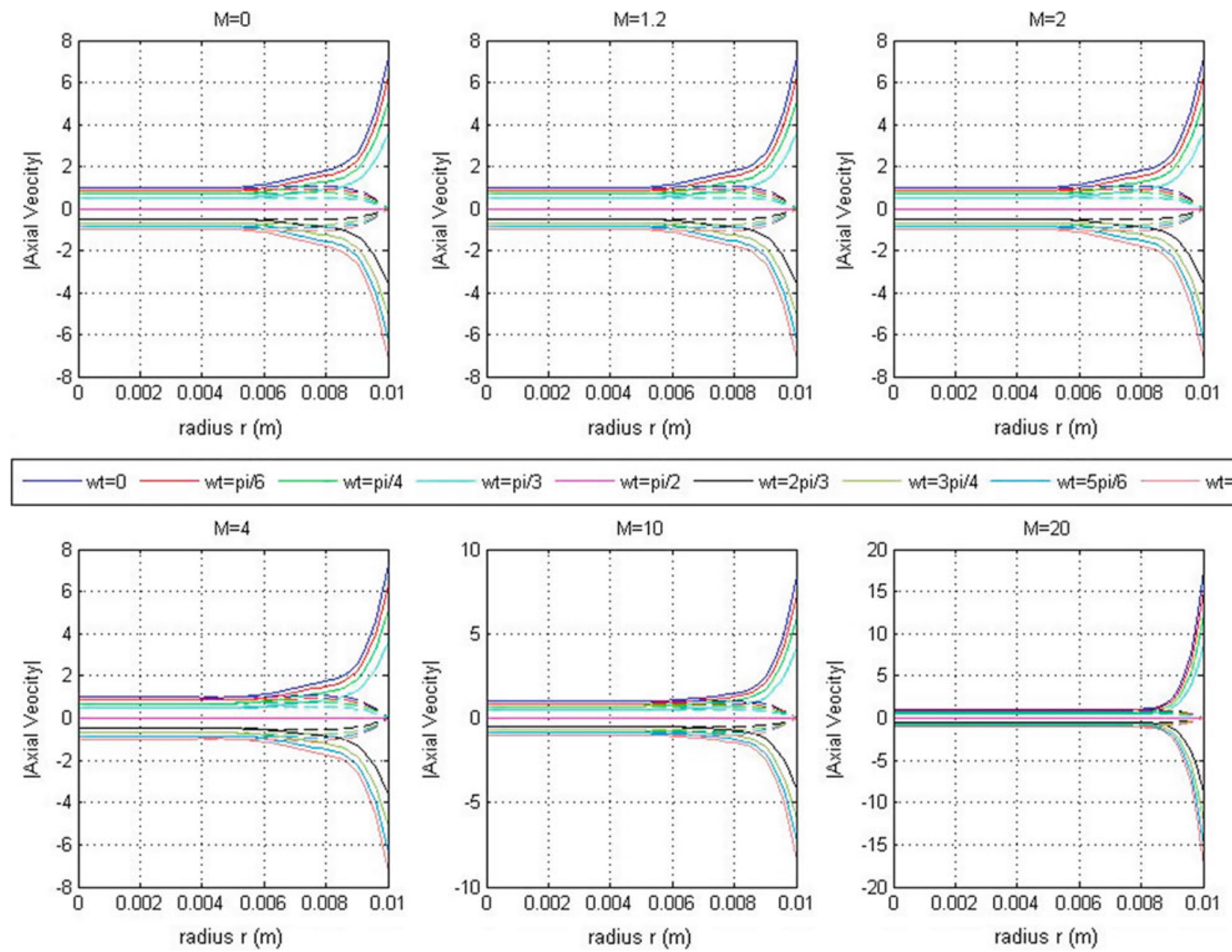

Fig. 5. The quantity $\Re\left(\left|\frac{u_{z}^{*}(r)}{u_{z}^{*}(0)}\right| e^{i \omega t}\right)$ is plotted for the deformable tube (solution of this paper - full lines) and for the rigid tube (solution of Sud et al. [27] - dotted lines), for fast waves (celerity $c_{2}$ ), and for different values of the Hartmann number $M$.

\subsection{Fluid velocities in the case $M=0$ : comparison with the solution of Atabek and Lew [37]}

In order to check the validity of our solution (Eqs. (40) and (41)), we have plotted on the same graphs our solutions, expressed in the particular case $M=0$, and the corresponding quantities given by Atabek and Lew [37]. This verification is presented in the Appendix.

\subsection{Influence of the Hartmann number $M$ on the non-dimensional fluid velocities}

The moduli of the amplitude of the non-dimensional axial velocity, $\left|\frac{u_{z}^{*}(r)}{C_{1}}\right|$, have been calculated using equation (40), for the two types of waves (Young waves, $c=c_{1}$, and Lamb waves, $\left.c=c_{2}\right)$. They are presented in Figure 2. The influence of $M$ becomes significant for values of $M$ higher than 4. As expected [32], the presence of an external magnetic field tends to flatten the velocity profile, and the profile becomes more and more different from Womersley's profile $(M=0)$. This is easy to understand, looking at the non-dimensional numbers $\alpha^{\prime}$ (defined in Eq. (19)) and $K$ (defined in Eq. (33)). When $M$ increases, $\alpha^{\prime}$ becomes more and more different from the classical Womersley's number: the effect of the magnetic field becomes preponderant, when compared to the pulsatility effect. Since the vessel is deformable, the velocity at the wall is not zero. However, the striking feature of Figure 2 is that, for the Lamb waves, huge velocity values are obtained at the wall. Such a result was previously obtained in the literature in the absence of a magnetic field (see for example, Figs. 3a and 3c in Jagielska et al. [39], or Fig. 18 in Jones et al. [45]). This is due to the fact that we did not introduce any kind of longitudinal constraint on the vessel wall. However, the effect is quite amplified in the presence of the magnetic field.

In Figure 3 are presented the moduli of the amplitude of the non-dimensional radial velocity. The quantity $\left|\frac{u_{r}^{*}(r)}{C_{1}} \frac{c}{\omega R}\right|$ is calculated from equation (41) and is plotted for the two types of waves (Young waves, $c=c_{1}$, and Lamb waves, $c=c_{2}$ ). As indicated in equation (41), the profiles of the radial velocity always start from zero at 

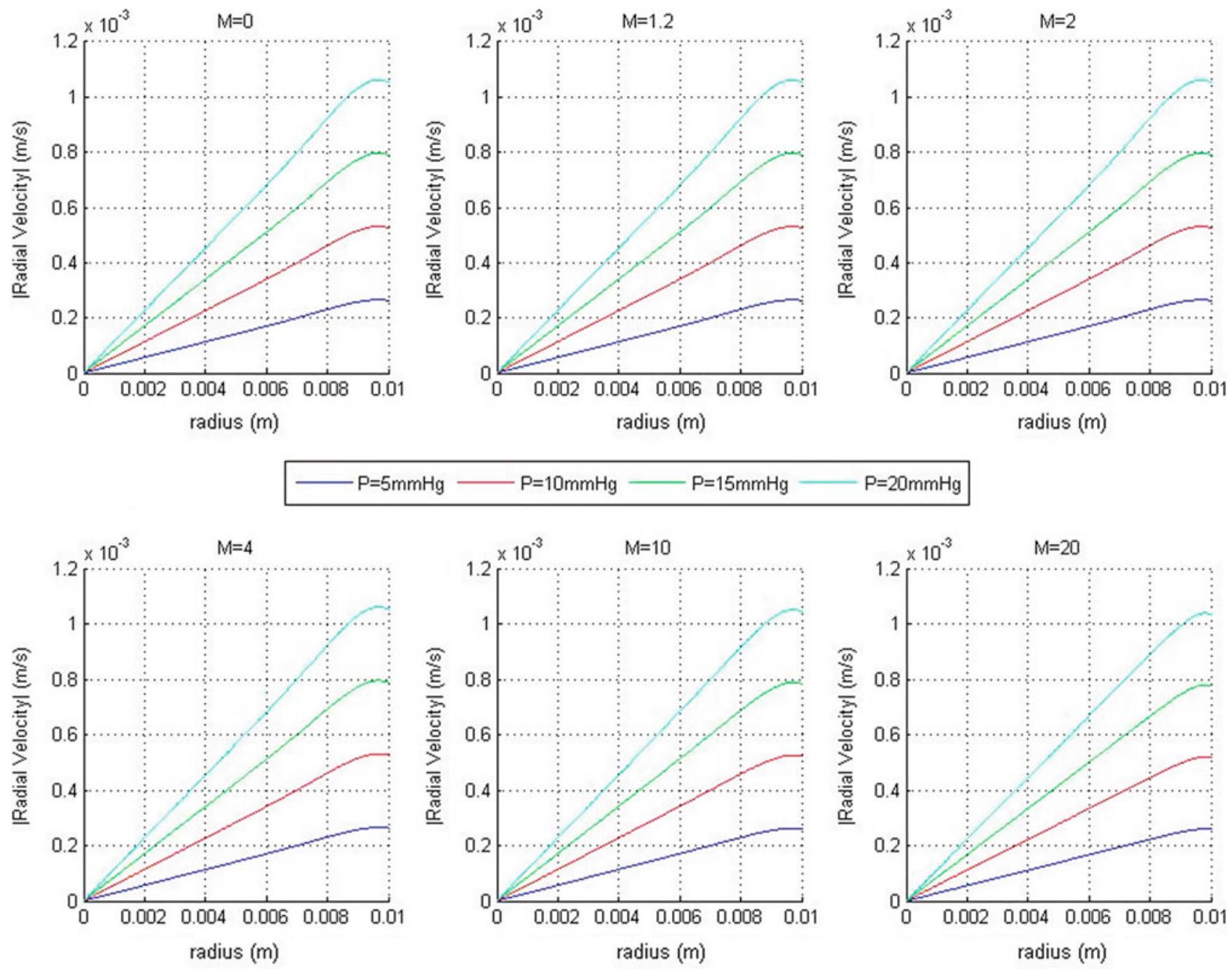

Fig. 6. Dimensional radial velocities (moduli) for $c=c_{1}$, for different values of the Hartmann number, and for different amplitudes of the pressure perturbation $(5 \mathrm{mmHg}, 10 \mathrm{mmHg}, 15 \mathrm{mmHg}, 20 \mathrm{mmHg}$ ).

the axis. The Hartmann number does not seem to have a great influence on these profiles in the case of the slow waves $\left(c=c_{1}\right)$, but in the case of Lamb waves, some differences appear near the vessel wall for $M$ higher than 4 . Our curves are quite similar to those of Figure $3 \mathrm{~d}$ in Jagielska et al. [39]. These authors studied the effect of some viscoelastic parameters of the tissues surrounding a vessel on the wave propagation. The Lorentz force we study in this paper opposes to the fluid motion in a manner that could be comparable to the external elastic and viscous forces they studied.

\subsection{Comparison deformable tube/rigid tube}

In order to see the impact of the deformability of the vessel, we have plotted $\Re\left(\left|\frac{u_{z}^{*}(r)}{u_{z}^{*}(0)}\right| e^{i \omega t}\right)$, for the deformable tube (Eq. (40) of this paper), and for the rigid tube [27].
The solution of Sud et al. [27] may be written in the form:

$$
\frac{u_{z}^{*}(r)}{u_{z}^{*}(0)}=\frac{J_{0}\left(\sqrt{-M^{2}-i \frac{\omega R^{2}}{\nu}}\right)-J_{0}\left(\sqrt{-M^{2}-i \frac{\omega R^{2}}{\nu}} \frac{r}{R}\right)}{J_{0}\left(\sqrt{-M^{2}-i \frac{\omega R^{2}}{\nu}}\right)-1}
$$

The results for the celerity $c_{1}$ (resp. $c_{2}$ ) are presented in Figure 4 (resp. Fig. 5). In the case of the rigid tube, the velocity at the wall is zero. This can more clearly be seen in Figure 5, but of course, it is also the case in Figure 4 . The $u_{z}^{*}(r)$ profiles for the deformable tube have been previously commented in Section 4.3. As expected, the main differences between the two solutions appear in the vicinity of the wall, but, for the slow waves, this difference remains small. 
A. Drochon: Sinusoïdal flow of blood in a cylindrical deformable vessel exposed to an external magnetic field
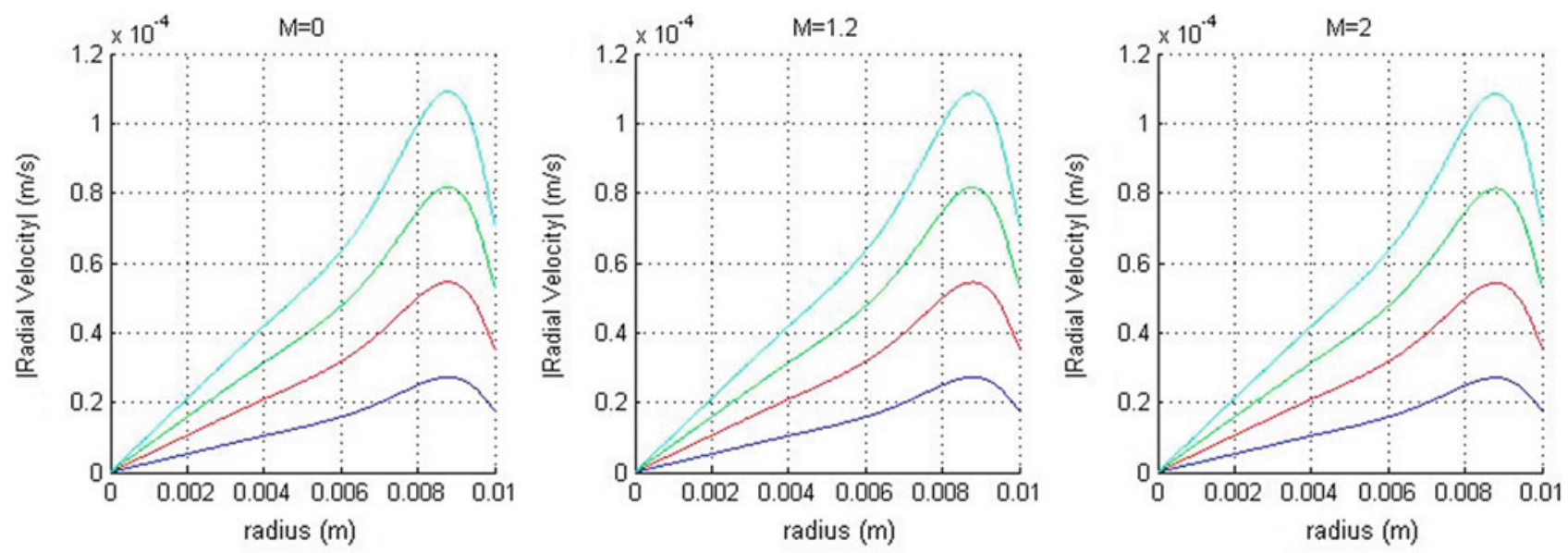

$-\mathrm{P}=5 \mathrm{mmHg}-\mathrm{P}=10 \mathrm{mmHg}-\mathrm{P}=15 \mathrm{mmHg}-\mathrm{P}=20 \mathrm{mmHg}$
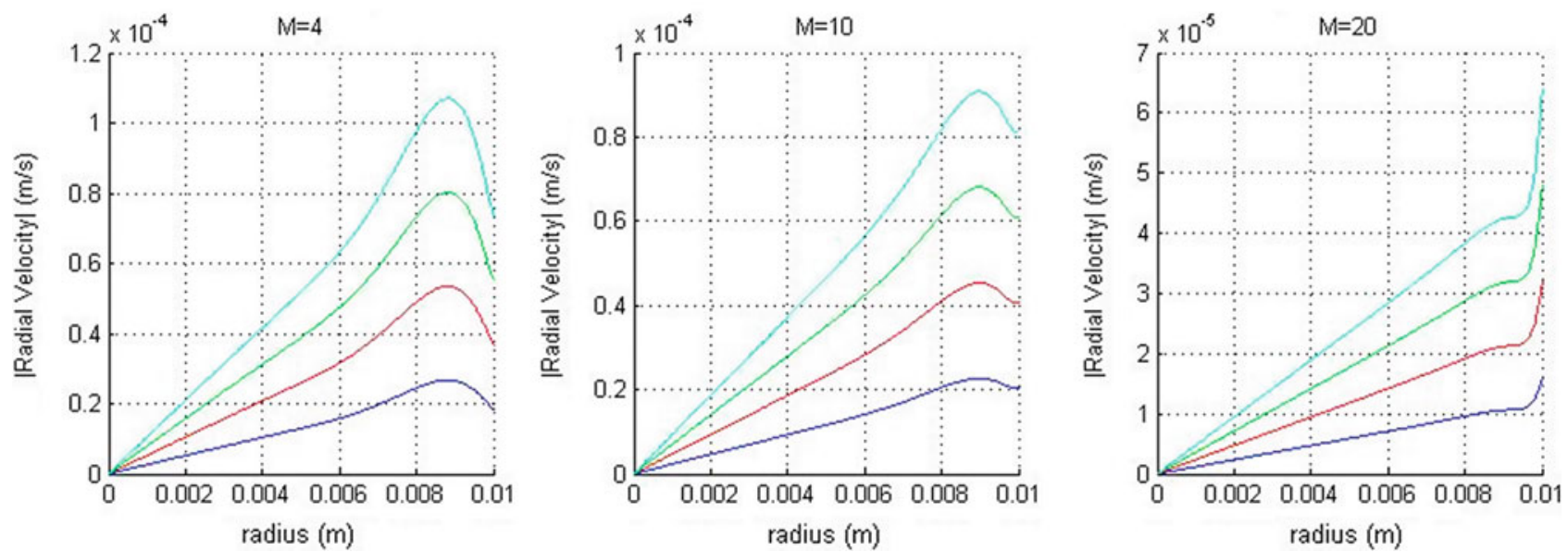

Fig. 7. Dimensional radial velocities (moduli) for $c=c_{2}$, for different values of the Hartmann number, and for different amplitudes of the pressure perturbation $(5 \mathrm{mmHg}, 10 \mathrm{mmHg}, 15 \mathrm{mmHg}, 20 \mathrm{mmHg}$ ).

\subsection{Influence of the amplitude of the pressure perturbation}

In order to evaluate the impact of the pressure perturbation on the fluid velocity, we have choosen four different levels of pressure perturbation: 5, 10, 15 and $20 \mathrm{mmHg}$. These numerical values fall in the range choosen by other authors. For example, Pontrelli and Tatone [46] took a pressure perturbation amplitude of $19.5 \mathrm{mmHg}$.

Using equation (16), and considering that, in the whole section of the vessel, $J_{0}\left(\frac{i \omega r}{c}\right) \approx 1$, it is possible to calculate the constant $C_{1}$ for any fixed value of $P^{*}(r)$, and for each celerity $c$. Considering then equation (37), it appears that the ratio $C_{2} / C_{1}$ does not itself depend on the pressure perturbation (it depends on $M$, on the celerity $c$ (who, itself, depends on $M$ ) and on the fluid and vessel characteristics). Consequently, it is easy to see from equations (40) and (41) that $u_{z}^{*}(r)$ and $u_{r}^{*}(r)$ will be directly proportional to $C_{1}$, i.e. to the pressure amplitude $P^{*}(r)$. This proportionality can be checked in the graphs of results (Figs. 6-9). As regards the axial and radial velocity profiles presented in Figures 6-9, they are similar to the non-dimensional ones that have been discussed in Section 4.3.

However, the results are now dimensional and it may be instructive to look at their order of magnitude:

- Moduli of the radial velocity:

For the Young waves (Fig. 6), we obtain $0.26 \mathrm{~mm} / \mathrm{s}$ at the wall for a pressure perturbation of $5 \mathrm{mmHg}$, and $1.05 \mathrm{~mm} / \mathrm{s}$ for a pressure perturbation of $20 \mathrm{mmHg}$. The influence of the Hartmann number on these values is not significant, even for $M=10$ or $M=20$.

For the Lamb waves (Fig. 7), the velocities at the wall are lower by a factor at least 10: from $17.8 \mu \mathrm{m} / \mathrm{s}$ to $71 \mu \mathrm{m} / \mathrm{s}$, in the case $M=0$. In the case $M=20$, the range of the values at the wall is: $16-64 \mu \mathrm{m} / \mathrm{s}$. But, as we previously mentioned in Section 4.3 , the velocity profile in the case $M=20$ is not the same as for lower values of the Hartmann number.

- Moduli of the axial velocity:

For the Young waves (Fig. 8), the order of magnitude of the velocities is now cm/s: from $6.8 \mathrm{~cm} / \mathrm{s}$ to $27 \mathrm{~cm} / \mathrm{s}$ on 

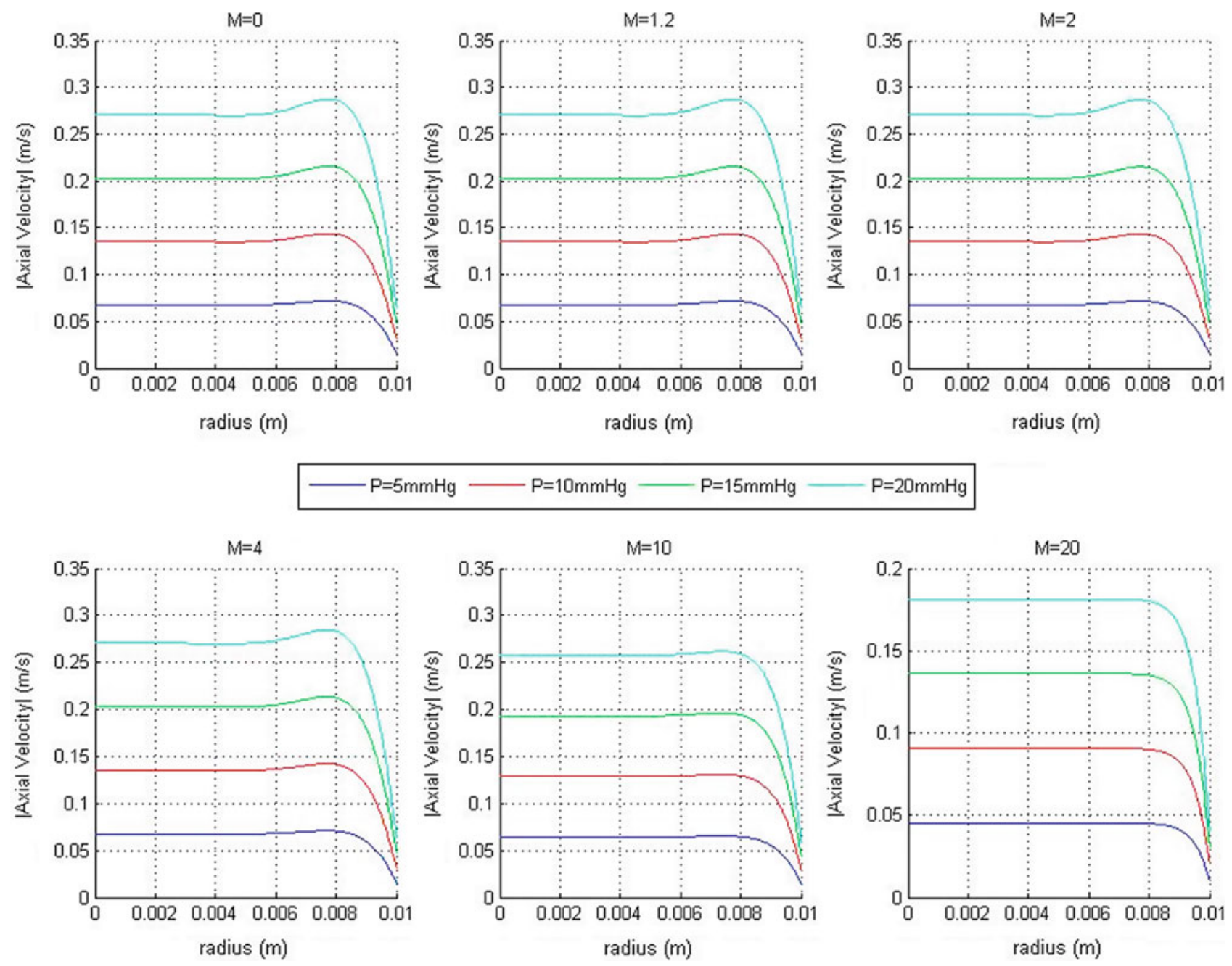

Fig. 8. Dimensional axial velocities (moduli) for $c=c_{1}$, for different values of the Hartmann number, and for different amplitudes of the pressure perturbation $(5 \mathrm{mmHg}, 10 \mathrm{mmHg}, 15 \mathrm{mmHg}, 20 \mathrm{mmHg}$ ).

the axis of the vessel and from $1.61 \mathrm{~cm} / \mathrm{s}$ to $6.4 \mathrm{~cm} / \mathrm{s}$ at the wall, in the case $M=0$. These values are reduced for high Hartmann numbers. For example, in the case $M=20$, we obtain values ranging from $4.5 \mathrm{~cm} / \mathrm{s}$ to $18 \mathrm{~cm} / \mathrm{s}$ on the vessel axis and from $1.01 \mathrm{~cm} / \mathrm{s}$ to $4.04 \mathrm{~cm} / \mathrm{s}$ at the wall. As in Section 4.3 and in our previous works $[28,32]$, we observe that the presence of an external magnetic field tends to reduce the blood flow and flatten the velocity profile and that the pulsatile character of the flow is dampened.

In the case of Lamb waves (Fig. 9), the velocities on the axis (from $2.06 \mathrm{~cm} / \mathrm{s}$ to $8.25 \mathrm{~cm} / \mathrm{s}$, in the case $M=0$ ) are lower than those of Young waves. However, they are much higher and not realistic at the wall (from $14.9 \mathrm{~cm} / \mathrm{s}$ to $59.6 \mathrm{~cm} / \mathrm{s}$ ). The velocities on the axis are reduced for high Hartmann numbers, but they are increased at the wall. For example, in the case $M=20$, we obtain values ranging from $0.93 \mathrm{~cm} / \mathrm{s}$ to $3.74 \mathrm{~cm} / \mathrm{s}$ on the vessel axis and from $16.38 \mathrm{~cm} / \mathrm{s}$ to $65.52 \mathrm{~cm} / \mathrm{s}$ at the wall.

Our values, especially the values for the axial velocity, are higher than those obtained by Jagielska et al. [39]. But these authors have choosen a very small pressure amplitude perturbation (1 $\mathrm{Pa})$ for their calculations. Zhang et al. [47] also considered the hypothesis that the surrounding tissue of a blood vessel can influence the biomechanics of the vessel. With an amplitude of $40 \mathrm{mmHg}$ for the pressure oscillations, they obtain axial fluid velocities of order $40 \mathrm{~cm} / \mathrm{s}$ and radial flow velocities of order $0.5 \mathrm{~mm} / \mathrm{s}$. These orders of magnitude are in agreement with ours (considering that our pressure amplitudes are lower than $40 \mathrm{mmHg}$ ). More recently, Warriner et al. [48] developed a visco-elastic model of arterial wall motion in pulsatile flow, motivated by the need for a realistic Doppler ultrasound clutter model. Their calculations provide values of the radial velocity at the wall around $0.1 \mathrm{~cm} / \mathrm{s}$ (same order of magnitude as us) and longitudinal velocities around $0.5 \mathrm{~cm} / \mathrm{s}$ (10 times smaller than our results). However, they implemented in their equations some additional terms that account for the wall viscoelasticity and for the wall tethering. This last point may explain the gap between their results and ours. Surprisingly, they do not mention the existence of the second type of waves (Lamb waves). They neither indicate very clearly the pressure amplitude they have choosen $(8 \mathrm{mmHg})$. 
A. Drochon: Sinusoïdal flow of blood in a cylindrical deformable vessel exposed to an external magnetic field
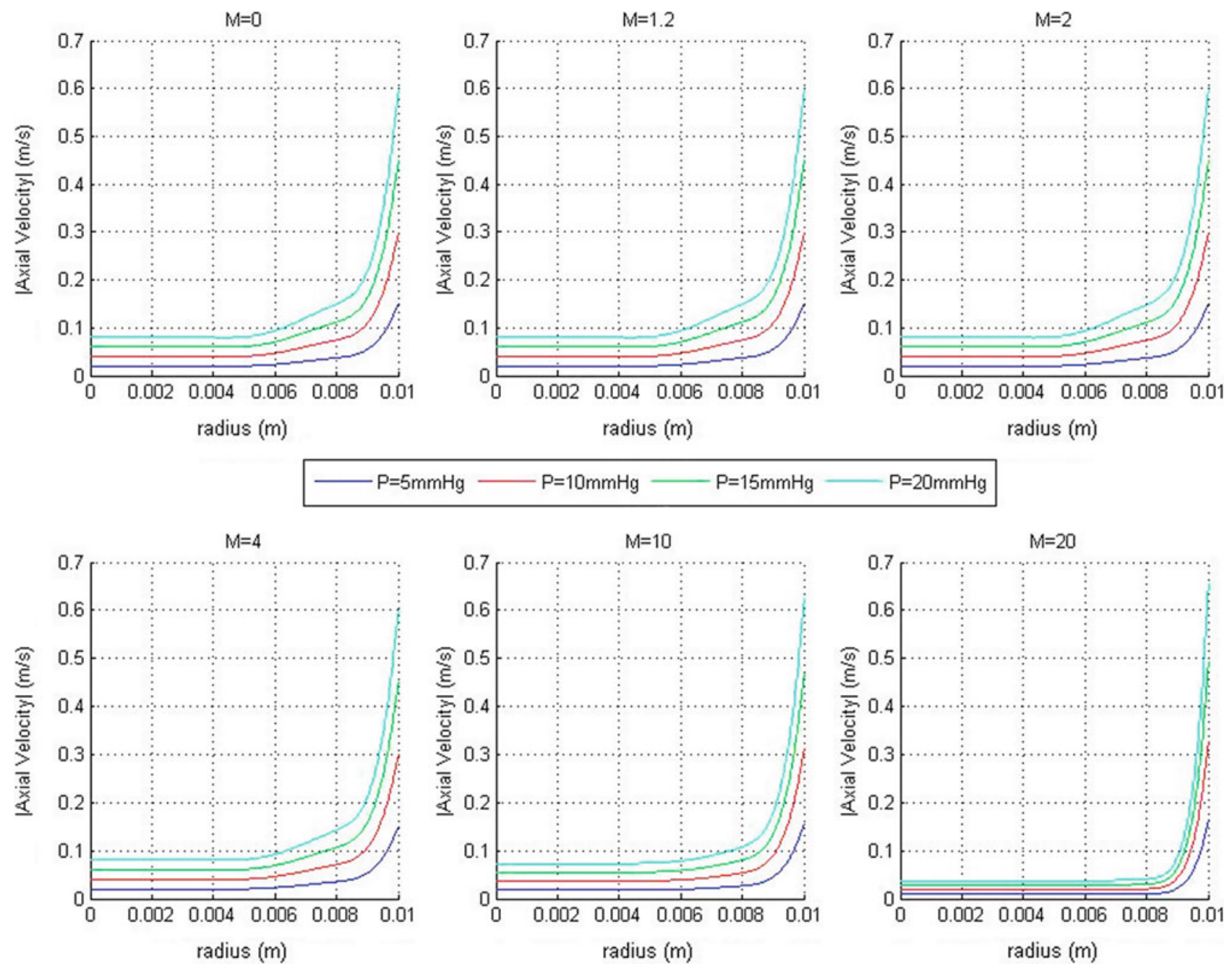

Fig. 9. Dimensional axial velocities (moduli) for $c=c_{2}$, for different values of the Hartmann number, and for different amplitudes of the pressure perturbation $(5 \mathrm{mmHg}, 10 \mathrm{mmHg}, 15 \mathrm{mmHg}, 20 \mathrm{mmHg}$ ).

Table 4. Values of the damping factor over a wavelength, $\exp (-2 \pi Y / X)$, for different Hartmann numbers and for the two modes of wave propagation.

\begin{tabular}{|c|c|c|c|c|c|c|}
\hline & $M=0$ & $\overline{M=1.2}$ & $M=2$ & $M=4$ & $M=10$ & $M=20$ \\
\hline $\begin{array}{c}\text { Young } \\
\text { waves } \\
\left(c=c_{1}\right) \\
\text { Lamb } \\
\text { waves }\end{array}$ & 0.8322 & 0.8149 & 0.7855 & 0.6604 & 0.21 & 0.0213 \\
\hline$\left(c=c_{2}\right)$ & 0.4933 & 0.4922 & 0.4900 & 0.4801 & 0.4175 & 0.2673 \\
\hline
\end{tabular}

\subsection{Wall displacements: propagation and damping of the waves}

In order to study the wave propagation and damping, we considered the wall displacements given by equation (42), for a pressure perturbation of $10 \mathrm{mmHg}$. The pulsatile term $\exp \left[i \omega\left(t-\frac{z}{c}\right)\right]$ may be developed as $\exp (i \omega t)$
Table 5. Values of the wavelengths corresponding to the celerities that are solution of our problem, for different Hartmann numbers and for the Young mode.

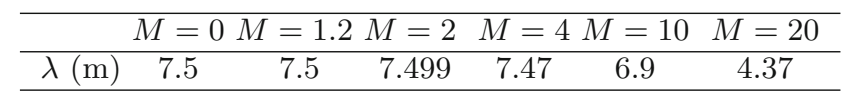

$\exp \left(-i \omega \frac{z}{c}\right)$. Using the notations of Womersley [34] and of Atabek and Lew [37], we express the complex roots of equation (32) as:

$$
\frac{c_{0}}{c}=X-i Y
$$

We then have: $\exp \left(-i \omega \frac{z}{c}\right)=\exp \left(-i \omega \frac{z X}{c_{0}}\right) \exp \left(-\omega z \frac{Y}{c_{0}}\right)$.

Inverting equation (49), we get: $\frac{c}{c_{0}} \approx \frac{1}{X}+i \frac{Y}{X^{2}}$, if $\frac{Y^{2}}{X^{2}} \ll 1$. The real part of $c$ is thus $c_{0} / X$; it is the propagation velocity and the associated wavelength is: $\lambda=\frac{c_{0}}{X} T$. 

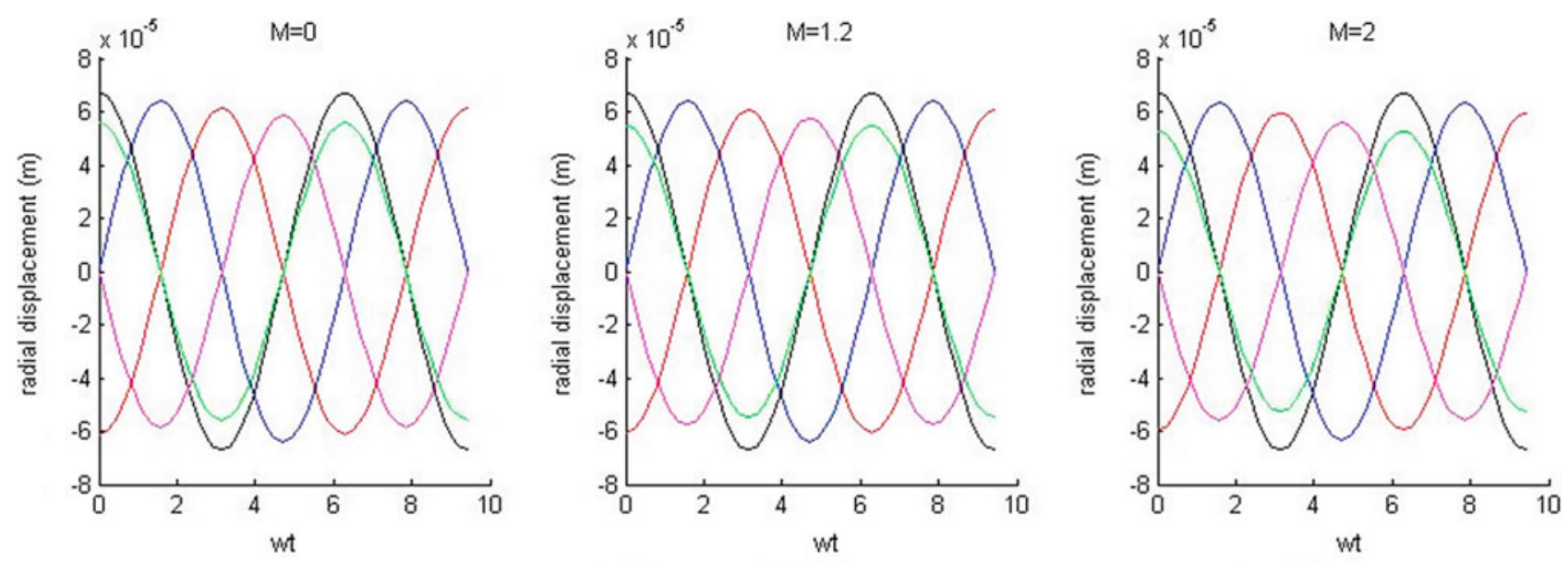

$\mathrm{z}=0-\mathrm{z}=\mathrm{ambda} / 4-\mathrm{z}=\mathrm{ambda} / 2-\mathrm{z}=3^{*} \mid \mathrm{ambda} / 4-\mathrm{z}-\mathrm{ambda}$
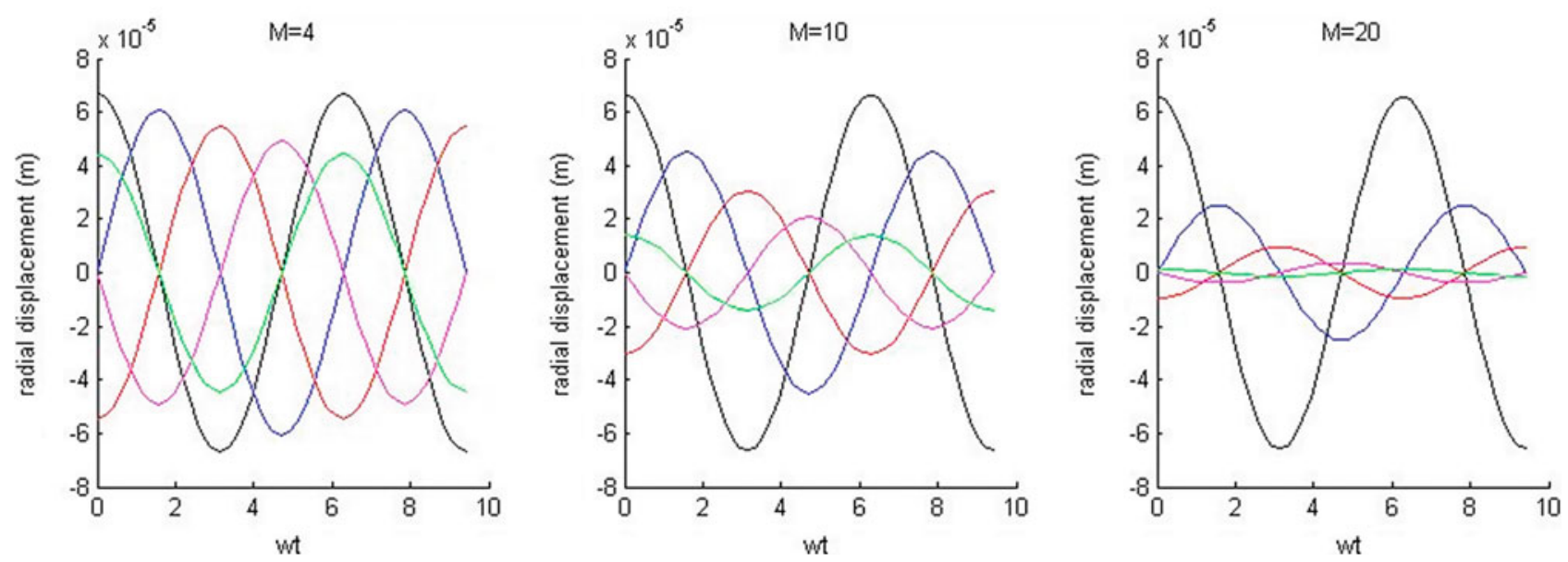

Fig. 10. Real part of $\left|C_{3}\right| \exp \left[i \omega\left(t-\frac{z}{c}\right)\right]$ (dimensional radial displacements) for $c=c_{1}$, for different values of the Hartmann number, and for different positions on the longitudinal axis.

Since $\omega=2 \pi / T$ ( $T=$ period of the pulse $)$, we finally get:

$$
\begin{aligned}
\exp \left[i \omega\left(t-\frac{z}{c}\right)\right]= & \exp (i \omega t) \\
& \times \exp \left(-2 \pi i \frac{z}{\lambda}\right) \exp \left(-2 \pi \frac{z}{\lambda} \frac{Y}{X}\right) .
\end{aligned}
$$

The last term of the right hand side of equation (50) is a real number and it gives the amount of damping per wavelength: when $z=\lambda$, this term equals $\exp (-2 \pi Y / X)$. This attenuation factor thus depends on the real and imaginary parts of the celerities, who, themselves, depend on the Hartmann number. This appears clearly in Table 4. In the case $M=0$, the damping effect is more pronounced for Lamb waves. However, for this type of waves, it is less sensitive to the magnetic field intensity than for the Young waves. One must also keep in mind that the wavelengths corresponding to the celerities that are solutions of our problem (celerities given in Tab. 2) are quite large when compared to the lengths of arteries. These wavelengths are given in Table 5 for the Young mode. Consequently, at typical places of palpation of the pulse wave amplitude (i.e., over a few centimeters or tens of centimeters), this damping effect will be hardly perceptible.

The real part of $\left|\frac{C_{3}}{C_{1}} C_{1}\right| \exp \left[i \omega\left(t-\frac{z}{c}\right)\right]=\left|C_{3}\right| \exp [i \omega(t-$ $\left.\frac{z}{c}\right)$ ] (radial displacements) is plotted in Figure 10 (Young waves) and in Figure 11 (Lamb waves), and the real part of $|C 4| \exp \left[i \omega\left(t-\frac{z}{c}\right)\right]$ (axial displacements) is plotted in Figure 12, for the Young waves. Similar graphs have been obtained for the axial displacements in the case of Lamb waves. They are not shown here, since the numerical values (up to $4 \mathrm{~cm}$ ) are not physiologic at all.

Looking at the larger ordinates in each graph, it is possible to estimate the relative proportion of the displacements: for Young waves, the ratio of radial displacements upon axial displacements is of order $1.6 \times 10^{-2}$ $(67 \mu \mathrm{m} / 4.13 \mathrm{~mm})$; for Lamb waves, the ratio of radial displacements upon axial displacements is of order $1.2 \times 10^{-4}$ $(4.5 \mu \mathrm{m} / 3.8 \mathrm{~cm})$; the ratio of radial displacements for slow waves upon radial displacements for fast waves is about $14.8(67 \mu \mathrm{m} / 4.5 \mu \mathrm{m})$ and the ratio of axial displacements 

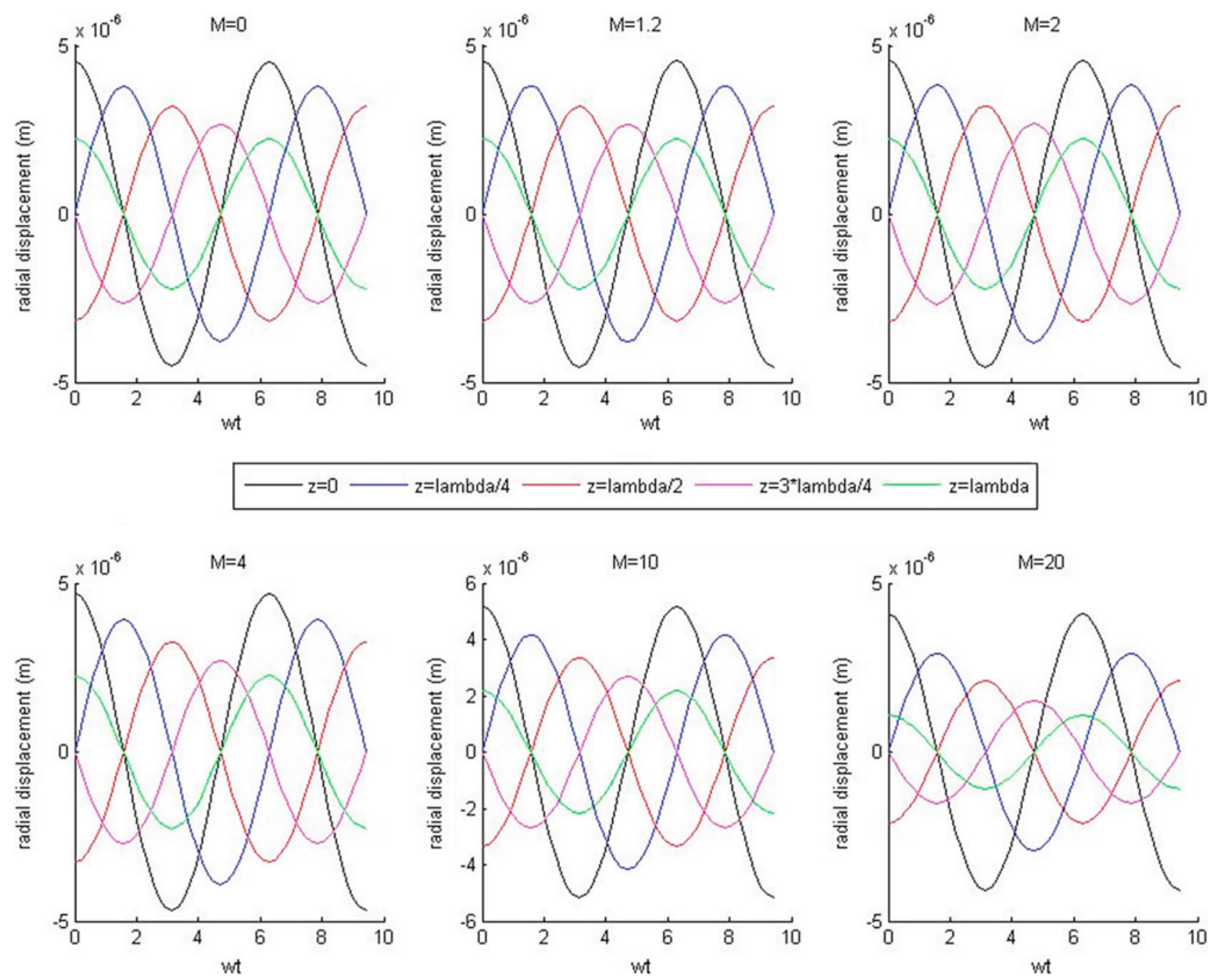

$z=l a m b d a / 4-z=l a m b d a / 2-z=3^{*} \mid a m b d a / 4-z=l a m b d a$
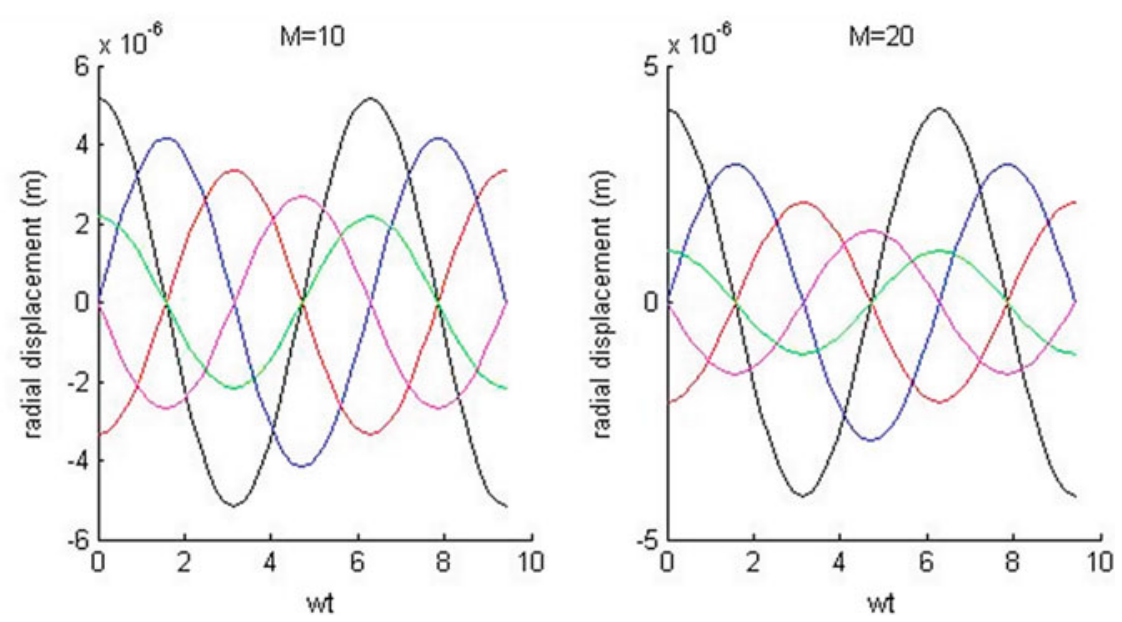

Fig. 11. Real part of $\left|C_{3}\right| \exp \left[i \omega\left(t-\frac{z}{c}\right)\right]$ (dimensional radial displacements) for $c=c_{2}$, for different values of the Hartmann number, and for different positions on the longitudinal axis.

for slow waves upon axial displacements for fast waves is about $0.11(4.13 \mathrm{~mm} / 3.8 \mathrm{~cm})$.

As expected, the Young mode has a more radial character and the Lamb mode has a more longitudinal character.

The literature papers addressing the question of vessel wall motion may be classified in two groups: (i) other models, and (ii) experimental works using magnetic resonance imaging or ultrasonic methods. As regards the results of other models, we note that Zhang et al. [47] obtain radial displacements of order $200 \mu \mathrm{m}$ in response to a $40 \mathrm{mmHg}$ pressure perturbation, which is in very good agreement with our data. Warriner et al. [48] provide data for the carotid artery: maximum radial distension from 100 to $200 \mu \mathrm{m}$ yielding percentages of diameter variation around $5-10 \%$ (which is somewhat higher than our $1 \%$ ). These authors also give results for the longitudinal wall motion (with wall tethering): about $200-300 \mu \mathrm{m}$, i.e., 10 times smaller than our $4 \mathrm{~mm}$, without wall tethering. More recently, Bukac and Canic [49] developed a mathematical model in order to capture both longitudinal and radial displacements of the wall. They report that the two components are of the same magnitude. Besides, direct measurements of the wall motion have been performed using non-invasive vascular ultrasound. For example, Reneman et al. [50] studied the loss of compliance of the carotid artery with age. They found that the carotid diameter (about $6 \mathrm{~mm}$ ) did not change very much for older persons, but the relative distensibility $(\Delta d / d)$ decreased from $13 \%$ to $5 \%$ ( $5 \%$ of $6 \mathrm{~mm}$ yields a diameter variation of $0.3 \mathrm{~mm}$, i.e., a radial displacement of $150 \mu \mathrm{m}$, which is not so far from our $70 \mu \mathrm{m})$.

Ramnarine et al. [51] obtained radial displacements of the carotid wall ranging from 500 to $100 \mu \mathrm{m}$, depending on the degree of stenosis of the artery (severe stenoses are associated with local wall stiffening and reduced deformation of the vessel). Cinthio et al. [52] measured longitudinal movements and diameter change of the common carotid artery of 10 healthy humans, using a new highresolution non-invasive ultrasonic method. They found longitudinal motions around $0.5 \mathrm{~mm}$, and diameter changes of the same magnitude (around $0.65 \mathrm{~mm}$ ). 
The European Physical Journal Applied Physics
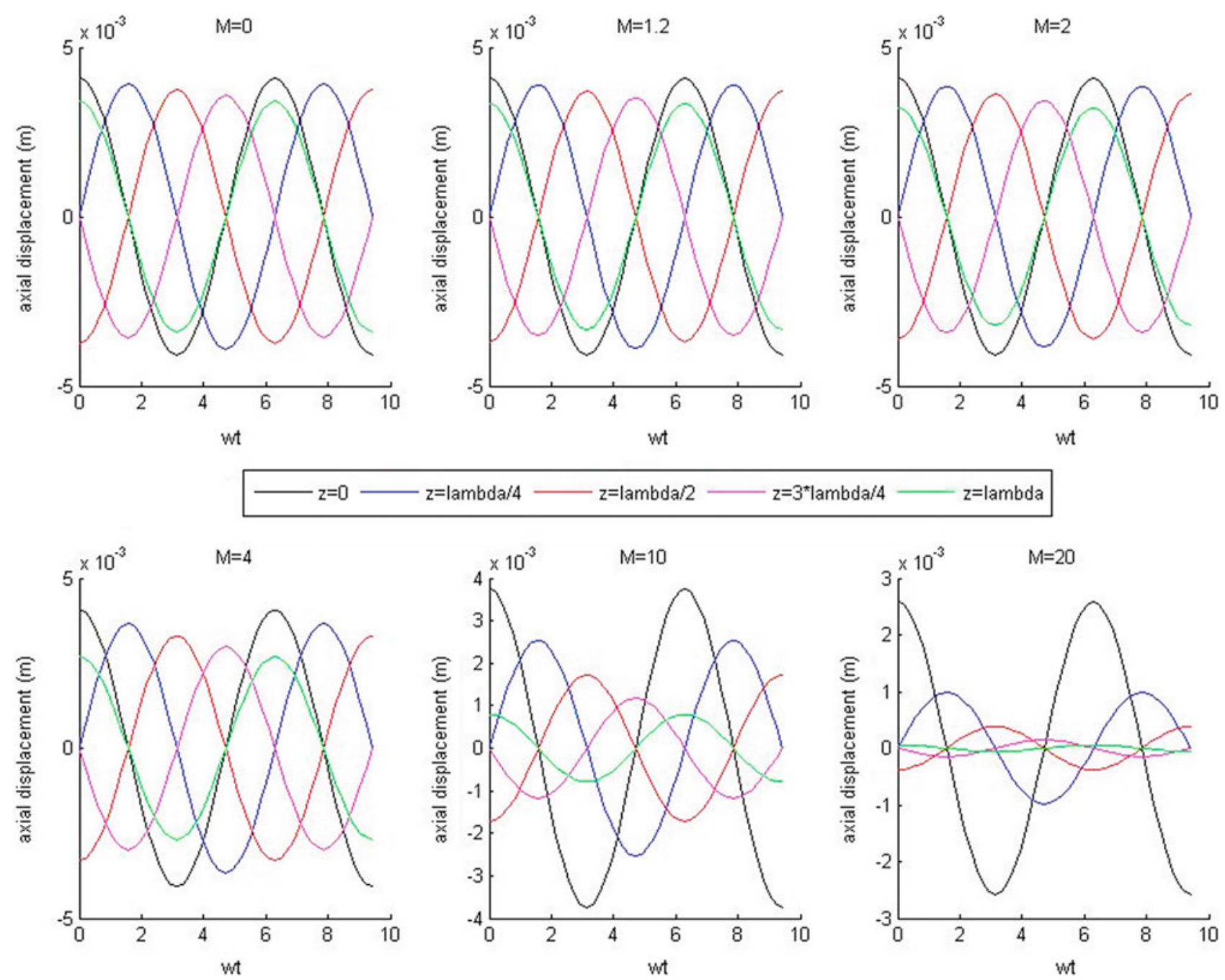

Fig. 12. Real part of $|C 4| \exp \left[i \omega\left(t-\frac{z}{c}\right)\right]$ (dimensional axial displacements) for $c=c_{1}$, for different values of the Hartmann number, and for different positions on the longitudinal axis.

They also performed the measurements for the aorta and obtained longitudinal movements as important as $1 \mathrm{~mm}$, and relative diameter changes about 9\%. However, the mechanisms responsible for these longitudinal movements are not clarified, although the shear force from the blood is probably of utmost importance. More recently, Bell et al. [53] performed magnetic resonance imaging in a large population and they measured aortic longitudinal and circumferential strains. They found that both types of strains remain lower than $10 \%$, and that they are not independent from each other (see their Fig. 1). Both studies [52,53] conclude that the measure of longitudinal wall displacements may become a novel marker of vascular hemodynamics and biology and, accordingly, of atherosclerosis and vascular disease.

\section{Conclusion}

In spite of the numerous restrictive assumptions, the solution presented here appears to describe reasonably well some important features of the magnetohydrodynamic flow of blood in an elastic vessel. It is demonstrated that the external magnetic field has an influence on the wave celerities in the fluid and in the vessel wall, on the fluid velocity profiles, and on the wall displacements. It tends to reduce the blood flow and flatten the velocity profile. The pulsatile character of the flow is also dampened. However, these effects become detectable for high values of the Hartmann number $(M>4$, corresponding to $B_{0}>36 \mathrm{~T}$ with numerical data pertaining to large human arteries) and remain negligible in the context of magnetic resonance imaging $\left(B_{0} \leq 3 \mathrm{~T}\right.$, or even $\left.7 \mathrm{~T}\right)$.

The author wants to thank several students who helped her for the graphs (Matlab tracings): Stephen Ozanne and Antoinette Benoit-de-Coignac, students of ENSEEIHT (Toulouse, France), and Laurine Redelsperger and Florian Devin, students of UTC (Compiègne, France). She also wants to thank several colleagues for constructive discussions on the topic of this paper: Dima Rodriguez (Abi-Abdallah) (from Université Paris Sud Orsay, France), Jean-Frédéric Gerbeau (from 
INRIA-Rocquencourt, France), Odette Fokapu, Vincent Robin, and Vincent Martin (from UTC, France).

\section{References}

1. D. Abi-Abdallah, V. Robin, A. Drochon, O. Fokapu, in IEEE Engineering in Medicine and Biology Society, Conference 200\%, pp. 1842-1845, http://ieeexplore.ieee.org

2. D. Abi-Abdallah, A. Drochon, V. Robin, O. Fokapu, Ann. Biomed. Eng. 35, 733 (2007)

3. G. Nijm, S. Swiryn, A. Larson, A. Sahakian, Med. Biol. Eng. Comput. 46, 729 (2008)

4. J. Krug, G. Rose, G. Clifford, J. Oster, J. Cardiovasc. Magn. Reson. 15, 104 (2013)

5. A. Kyriakou, E. Neufeld, D. Szczerba, W. Kainz, R. Luechinger, S. Kozerke, R. Mc Gregor, N. Kuster, Physiol. Meas. 33, 117 (2012)

6. T. Frauenrath, K. Fuchs, M. Dieringer, C. Ozerdem, N. Patel, W. Renz, A. Greiser, T. Elgeti, T. Niendorf, J. Magn. Reson. Imaging 36, 364 (2012)

7. T. Niendorf, A. Graessl, Ch. Thalhammer, M. Dieringer, O. Kraus, D. Santoro, K. Fuchs, F. Hezel, S. Waiczies, B. Ittermann, L. Winter, J. Magn. Reson. 229, 208 (2013)

8. E. Laffon, R. Marthan, M. Montaudon, V. Latrabe, F. Laurent, D. Ducassou, J. Magn. Reson. Imaging 21, $53(2005)$

9. L. Joly, C. Perret-Guillaume, A. Kearney-Schwartz, P. Salvi, D. Mandry, P.Y. Marie, G. Karcher, P. Rossignol, F. Zannad, A. Benetos, Hypertension 54, 421 (2009)

10. E.S. Ibrahim, K. Johnson, A. Miller, J. Shaffer, R. White, J. Cardiovasc. Magn. Reson. 12, 26 (2010)

11. M. Markl, W. Wallis, S. Brendecke, J. Simon, A. Frydrychowicz, A. Harloff, Magn. Reson. Med. 63, 1575 (2010)

12. A. Pfenniger, L. Wickramarathna, R. Vogel, V. Koch, Med. Eng. Phys. 35, 1256 (2013)

13. A. Pfenniger, D. Obrist, A. Stahel, V. Koch, R. Vogel, Med. Biol. Eng. Comput. 51, 741 (2013)

14. D. Jia, J. Liu, Y. Zhou, Phys. Lett. A 373, 1305 (2009)

15. M. Poirier-Quinot, G. Frasca, C. Wilhelm, N. Luciani, J.C. Ginefri, L. Darasse, D. Letourneur, C. Le Visage, F. Gazeau, Tissue Eng. Part C Methods 16, 185 (2010)

16. H. Xu, S. Othman, R. Magin, J. Biosci. Bioeng. 106, 515 (2008)

17. H. Perea, J. Aigner, U. Hopfner, E. Wintermantel, Cells Tissues Organs 183, 156 (2006)

18. H. Perea, J. Aigner, J.T. Heverhagen, U. Hopfner, E. Wintermantel, J. Tissue Eng. Regen. Med. 1, 318 (2007)

19. A. Ito, K. Ino, M. Hayashida, T. Kobayashi, H. Matsunuma, H. Kagami, M. Ueda, H. Honda, Tissue Eng. 11, 1553 (2005)

20. P. Babinec, A. Krafcik, M. Babincova, J. Rosenecker, Med. Biol. Eng. Comput. 48, 745 (2010)

21. J. Riegler, K. Lau, A. Garcia-Prieto, A. Price, T. Richards, Q. Pankhurst, M. Lythgoe, Med. Phys. 38, 3932 (2011)
22. G. Ciofani, C. Riggio, V. Raffa, A. Menciassi, A. Cuschieri, Med. Hypotheses 73, 80 (2009)

23. A. Morega, A. Dobre, M. Morega, Rev. Roum. Sci. Tech. - Electrotech et Energ. 56, 199 (2011)

24. R. Gold, J. Fluid Mech. 13, 505 (1962)

25. V. Vardanyan, Biofizika 18, 515 (1973)

26. J. Keltner, M. Roots, P. Brakeman, T. Budinger, Magn. Reson. Med. 16, 139 (1990)

27. V. Sud, P. Suri, R. Mishra, Studia Biophysica 46, 163 (1974)

28. D. Abi-Abdallah, A. Drochon, V. Robin, O. Fokapu, Comput. Methods Biomech. Biomed. Eng. 12, 445 (2009)

29. Y. Kinouchi, H. Yamaguchi, T.S. Tenforde, Bioelectromagnetics 17, 21 (1996)

30. V. Martin, A. Drochon, O. Fokapu, J.F. Gerbeau, Phys. Med. Biol. 57, 1 (2012)

31. G. Shit, Model. Simul. Engin. 2013, 758748 (2013)

32. D. Abi-Abdallah, A. Drochon, V. Robin, O. Fokapu, Eur. Phys. J. Appl. Phys. 45, 11301 (2009)

33. D. Sankar, U. Lee, A.I.M. Ismail, Int. J. Non-Linear Sci. Numer. Simul. 14, 195 (2013)

34. J. Womersley, J. Phil. Mag. 46, 199 (1955)

35. D. Abi-Abdallah, A. Drochon, V. Robin, O. Fokapu, J. Biomech. 41, S269 (2008)

36. R. Cox, Biophys. J. 8, 691 (1968)

37. H. Atabek, H. Lew, Biophys. J. 6, 481 (1966)

38. P. Flaud, I. Rogova, Biomécanique des fluides et des tissus, edited by M. Jaffrin, F. Goubel (Ed. Masson, 1998)

39. K. Jagielska, D. Trzupek, M. Lepers, A. Pelc, P. Zielinski, Phys. Rev. E 76, 066304 (2007)

40. G. Kuiken, J. Fluid Mech. 141, 289 (1984)

41. Y. Ma, C.-O. Ng, Phys. Fluids 21, 051901 (2009)

42. J. Wang, K. Parker, J. Biomech. 37, 457 (2004)

43. B. Bolster, E. Atalar, C. Hardy, E. McVeigh, J. Magn. Reson. Imaging 8, 878 (1998)

44. S. Roes, R. Dehnavi, J. Westenberg, H. Lamb, B. Mertens, J. Tamsma, A. De Roos, Diabetes Care 31, 1442 (2008)

45. E. Jones, M. Anliker, I.D. Chang, Biophys. J. 11, 1085 (1971)

46. G. Pontrelli, A. Tatone, Eur. J. Mech. B: Fluids 25, 987 (2006)

47. W. Zhang, C. Herrera, S. Atluri, G. Kassab, J. Biomech. Eng. 126, 760 (2004)

48. R. Warriner, K. Johnston, R. Cobbold, Physiol. Meas. 29, 157 (2008)

49. M. Bukac, S. Canic, Math. Biosci. Eng. 10, 295 (2013)

50. R. Reneman, J. Meinders, A. Hoeks, Eur. Heart J. 26, 960 (2005)

51. K. Ramnarine, T. Hartshorne, Y. Sensier, M. Naylor, J. Walker, A. Naylor, R. Panerai, D. Evans, Cardiovasc. Ultrasound 1, 1 (2003)

52. M. Cinthio, A. Ahlgren, J. Bergkvist, T. Jansson, H. Persson, K. Lindstrom, Am. J. Physiol: Heart Circ. Physiol. 291, H394 (2006)

53. V. Bell, W. Mitchell, S. Sigurosson, J. Westenberg, J. Gotal, A. Torjesen, T. Aspelund, L. Launer, A. De Roos, V. Gudnason, T. Harris, G. Mitchell, J. American Heart Association 3, e001536 (2014) 


\section{Appendix}

In order to check the validity of our solution (Eqs. (40) and (41)), we have plotted on the same graphs our solutions, expressed in the particular case $M=0$, and the corresponding quantities given by Atabek and Lew [37].

In Atabek and Lew [37], our term $u_{z}^{*}(r) / C_{1}$ is given by:

$$
\frac{u_{z}^{*}(r)}{C_{1}}=1+m \frac{J_{0}\left(\alpha_{0} \frac{r}{R}\right)}{J_{0}\left(\alpha_{0}\right)},
$$

and our term $u_{r}^{*}(r) / C_{1}$ is given by:

$$
\frac{u_{r}^{*}(r)}{C_{1}}=\frac{i \omega R}{c}\left[\frac{r}{2 R}+m \frac{J_{1}\left(\alpha_{0} \frac{r}{R}\right)}{\alpha_{0} J_{0}\left(\alpha_{0}\right)}\right]
$$

where: $m=\frac{3 R \rho c^{2}}{2 E h\left(F_{10}-1\right)}$ and $\alpha_{0}=i^{3 / 2} R \sqrt{\frac{\omega}{\nu}}$ (their constant $A$ is equivalent to $\rho c C_{1}$ in our notations).

Equations (A1) and (A2) have been both plotted for the two types of waves (celerity $c_{1}$, and $c_{2}$ ). Only one illustrative graph is shown here (Fig. A1), but a perfect fit between the two solutions has been also obtained in the three other graphs.

This result is not surprising if we consider the limiting case $M=0$ in our equations: we have $K=1$, and since $\left(\omega \nu / c^{2}\right)$ is very small, the ratio $C_{2} / C_{1}$ given by equation (37) in this

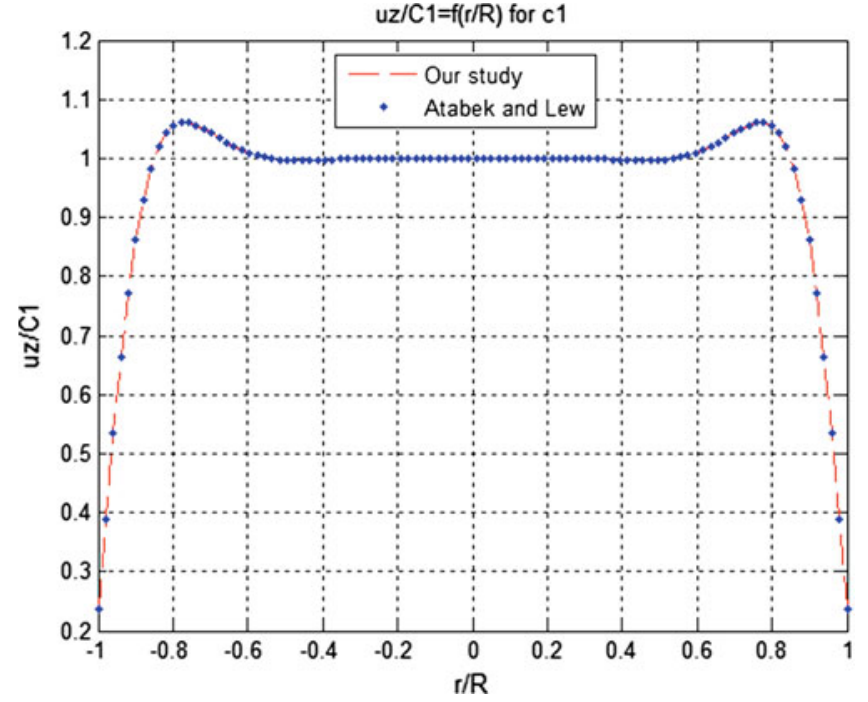

Fig. A1. Real part of the non-dimensional axial velocity $u_{z}^{*}(r) / C_{1}$ for the celerity $c=c_{1}$ (Young mode). Comparison between our solution in the particular case $M=0$ and the solution of Atabek and Lew [37].

paper turns out to be the " $m$ " of Atabek and Lew [37] (since, in that case, our quantity $F_{10}^{\prime}$ reduces to their " $F_{10}$ "). 